\title{
HIGH-ENERGY X-RAYS: A TOOL FOR ADVANCED BULK INVESTIGATIONS IN MATERIALS SCIENCE AND PHYSICS
}

\author{
KLAUS-DIETER LISS ${ }^{\mathrm{a}, *}$, ARNO BARTELS ${ }^{\mathrm{a}}$, \\ ANDREAS SCHREYER $^{\mathrm{b}}$ and HELMUT CLEMENS ${ }^{\mathrm{c}}$ \\ a Arbeitsbereich für Werkstoffphysik und-technologie, \\ Technische Universität Hamburg-Harburg, \\ Eißendorferstraße 40, D-21073 Hamburg, Germany; \\ ${ }^{\mathrm{b}}$ Division for Neutron and X-Ray Scattering, GKSS-Research Center, \\ Max-Planck-Straße 1, D21494 Geesthacht, Germany; \\ ${ }^{\mathrm{c}}$ Institut für Metallkunde und Werkstoffprüfung, Montanuniversität, \\ Franz-Josef-Straße 18, A-8700 Leoben, Austria
}

(Received 1 September 2003)

\begin{abstract}
High-energy X-rays between $30 \mathrm{keV}$ and $1 \mathrm{MeV}$, such as provided by modern synchrotron radiation sources as the ESRF and HASYLAB, bear the advantage of high penetration into most materials. Even heavy element compositions can be accessed in their volume. The range of applications is huge and spreads from nuclear spectroscopy to the characterization of metal extrusion under industrial conditions. This article compiles an overview over the most common instrumental diffraction techniques.

Modern two-dimensional detectors are used to obtain rapid overviews in reciprocal space. For example, diffuse scattering investigations benefit from the very flat Ewald sphere as compared to low energies, which allow mapping of several Brillouin zones within one single shot. Diffraction profiles from liquids or amorphous materials can be recorded easily. For materials science purposes, whole sets of Debye-Scherrer rings are registered onto the detector, their diameters and eccentricities or their intensity distribution along the rings relating to anisotropic strain or texture measurements, respectively. At this point we stress the resolution of this technique which has to be carefully taken into account when working on a second generation synchrotron source.

Energy-dispersive studies of local residual strain can be studied by a dedicated three-circle diffractometer which allows accurately to adjust the scattering angle from a defined gauge volume.

Triple axis diffractometry and reciprocal space mapping is introduced and can be employed for highest resolution purposes on single crystal characterization, even under heavy and dense sample environments. Thus, the perfection of single crystals can be mapped and strain fields and superstructures as introduced by the modulation from ultrasonic waves into crystals or epitaxially grown $\mathrm{Si} / \mathrm{Ge}$ layers can be investigated in detail. Phase transitions as magnetic ordering can be studied directly or through its coupling to the crystal lattice. Time resolved studies are performed stroboscopically from a sub-nanosecond to a second time scale.

The combination of these techniques is a strong issue for the construction and development of future instruments.
\end{abstract}

Keywords: High-energy X-rays; Two-dimensional diffraction; Triple-axes diffractometry

*Corresponding author. E-mail: liss@kdliss.de 


\section{INTRODUCTION}

The applications of high-energy X-rays have been continuously developed with the upcome of dedicated synchrotron sources one decade ago and benefit from more and more popularity. They have been employed by the most different techniques concerning different questions, as the Compton spectroscopy to measure the momentum-distributions of bound electrons (Isaacs et al., 1999; Suorti et al., 2001) or the atomic and nuclear spectroscopy to access high-energy levels (Bikit et al., 1987; Materna et al., 1999; Schnier, 2002). High-resolution triple crystal diffractometry is pursued to investigate phase transitions in single crystals such as magnetic or charge ordering and structural changes (Strempfer et al., 1996, 1997; Chatterji et al., 1998; Wilkins et al., 2000; Bastie et al., 2003; Hatton et al., 2003; Liss et al., 2003; Miclaus and Goorsky, 2003) or to analyze artificial superstructures or tiny strain gradients as in optical band-gap structures (Liss et al., 1998b) or ultrasonically excited crystals (Liss et al., 1997a,b, 1998a). Both white beam and monochromatic beam technologies can be employed to study residual strains and textures in polycrystalline samples for materials science purposes (Reimers et al., 1998, 1999; Pyzalla et al., 1999,2000a,b,c, 2001; Withers et al., 2002; Brokmeier et al., 2003; Wcislak et al., 2003). Imaging methods and thus computed tomography are possible and, nowadays, are being developed rapidly (Jolie et al., 1999; Materna et al., 1999; Lame et al., 2003).

Historically, high-energy X-rays were obtained from radioactive sources like ${ }^{137} \mathrm{Cs}$, ${ }^{197} \mathrm{Au}$ or ${ }^{192} \mathrm{Ir}$ with characteristic nuclear $\gamma$ radiation at 660,412 and $317 \mathrm{keV}$, respectively (Schneider, 1974; Jauch and Palmer, 1999). They have been employed for the characterization of single crystalline materials as beryllium (May et al., 1995), Heusler alloys (Freund et al., 1983), intercalated graphite (Fischer et al., 1991) vapor grown materials as $\mathrm{HgI}_{2}$ (Rossberg et al., 1994), shear creep in nickel based super alloys (Maniguet et al., 1995) and much more. Even though unique experiments can be performed by $\gamma$-ray sources (Jauch, 2001), high-energy X-ray tubes are available with a continuous spectrum up to several hundred $\mathrm{keV}$ replacing these nuclear sources, which were too difficult to handle (Bastie et al., 2000). High brilliance with energies well above $100 \mathrm{keV}$, however, are obtained at synchrotron radiation sources with the first developments at the DORIS and later the PETRA storage rings at HASYLAB in Hamburg, Germany (Hahn et al., 1997). Beyond the third generation sources, the European Synchrotron Radiation Facility (ESRF) was planned to come up with a dedicated high-energy beamline ID15 which is in operation since mid 1994 (Tschentscher and Suortti, 1998). At that time, the user community was small and applications had to be developed in the diverse fields of physics, chemistry, life sciences and materials science. High-energy programs are developed sometimes with huge effort in order to reach a $100 \mathrm{keV}$ with a lower-energy electron beam such as at the Pohang Light Source (Sohn et al., 2001). Presently, dedicated beamlines are planned to be constructed by traditional institutes for materials science employing so far neutron radiation like the GKSS in Geesthacht near Hamburg (Beckmann et al., 2003; Schreyer et al., 2003). Actually, GKSS completely refurbishes a new, dedicated beamline HARWI-II at DORIS and is involved in defining beamline projects at the future PETRA-III, a project delivering higher emittance and thus brilliances than the ESRF nowadays. Among the three high-energy third generation sources ESRF, APS in the USA and SPRING-8 in Japan the beamline ID15A at ESRF offers the highest X-ray energies 
in the range between $30 \mathrm{keV}$ and $1 \mathrm{MeV}$, which is typically one order of magnitude higher than that on most other beamlines at the same storage ring.

\section{HIGH-ENERGY X-RAYS}

The main advantage of high-energy X-rays is their penetration into matter which allows for the investigation of bulk material. The attenuation lengths $\tau_{0}$ for an intensity decreases to $e^{-1}$ for the elements of the fifth group of the periodic table, standing as a typical example for all the periods, are manifested in Fig. 1. The energy range from $30 \mathrm{keV}$ to $1 \mathrm{MeV}$ accessible on a typical high-energy beamline is marked in light gray while most diffraction experiments take place between 80 and $500 \mathrm{keV}$. Generally, attenuation is marked by three regions namely photo absorption which strongly decreases with the third power of the photon energy and shows the characteristic absorption edges, the Compton scattering which depends weakly on energy and at last pair production which onsets at $1022 \mathrm{keV}$ well above the energy range we are interested in. For the light elements, only Compton scattering occurs, making them favorable for beam attenuators without modifying the spectral distribution too much. Samples of many centimeters thickness can be accessed. Note that silicon becomes even more transparent than diamond which makes this cheap and perfect material applicable for monochromators for multiple beam side-stations, such as realized on ID15A (Tschentscher and Suortti, 1998). For the heaviest elements photo absorption

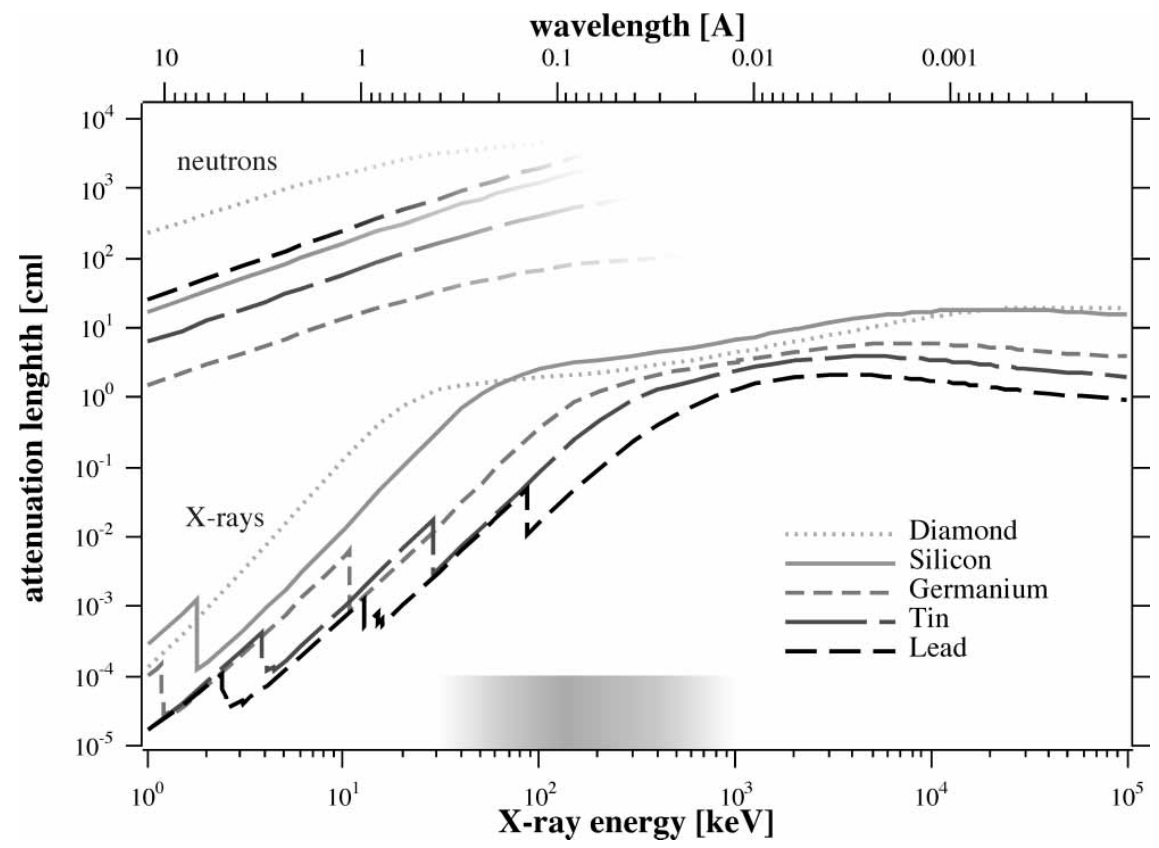

FIGURE 1 Attenuation lengths of the elements of the 5th group, representing the typical behavior for the different periods. The three attenuation processes, photo absorption, Compton scattering and pair production are distinguishable by their different slopes. The gray shaded area shows the energy range accessible at ID15A. Note, that silicon is more transparent than diamond in this energy range which allows to take off monochromatic beam for two further side stations while the transmitted beam continues to the main station. 
does not cease but volumes of thickness in the millimeter range are accessible. The $K$ absorption edges increase with the atomic number $Z$ and the one for uranium lies at $115.6 \mathrm{keV}$. They can be useful for imaging methods in materials and environmental research (Materna et al., 1999). Only neutron radiation may penetrate deeper and shows no continuous dependency of the penetration on the atomic number $Z$.

Attenuation is one of the primary parameters when envisaging a bulk sample measurement. The attenuation length $\tau_{0}$ can be calculated from the mass absorption coefficient $\Sigma$ which is listed in literature (Berger et al., 1998) by

$$
\tau_{0}=\frac{1}{\Sigma \cdot \rho}
$$

where $\rho$ stands for the density of the material.

\section{PHOTON FLUX}

The photon fluxes of the high-energy sources at ESRF and HASYLAB are compared in Fig. 2. They are calculated for a pinhole of $1 \times 1 \mathrm{~mm}^{2}$ which is positioned approximately at the monochromator or sample position of the beamline. ID15 has two optional sources, the Asymmetric Multi Pole Wiggler (AMPW) (Tschentscher and Suortti, 1998) and a new 3 Tesla device W3T (Chavanne et al., 1999). With only 7

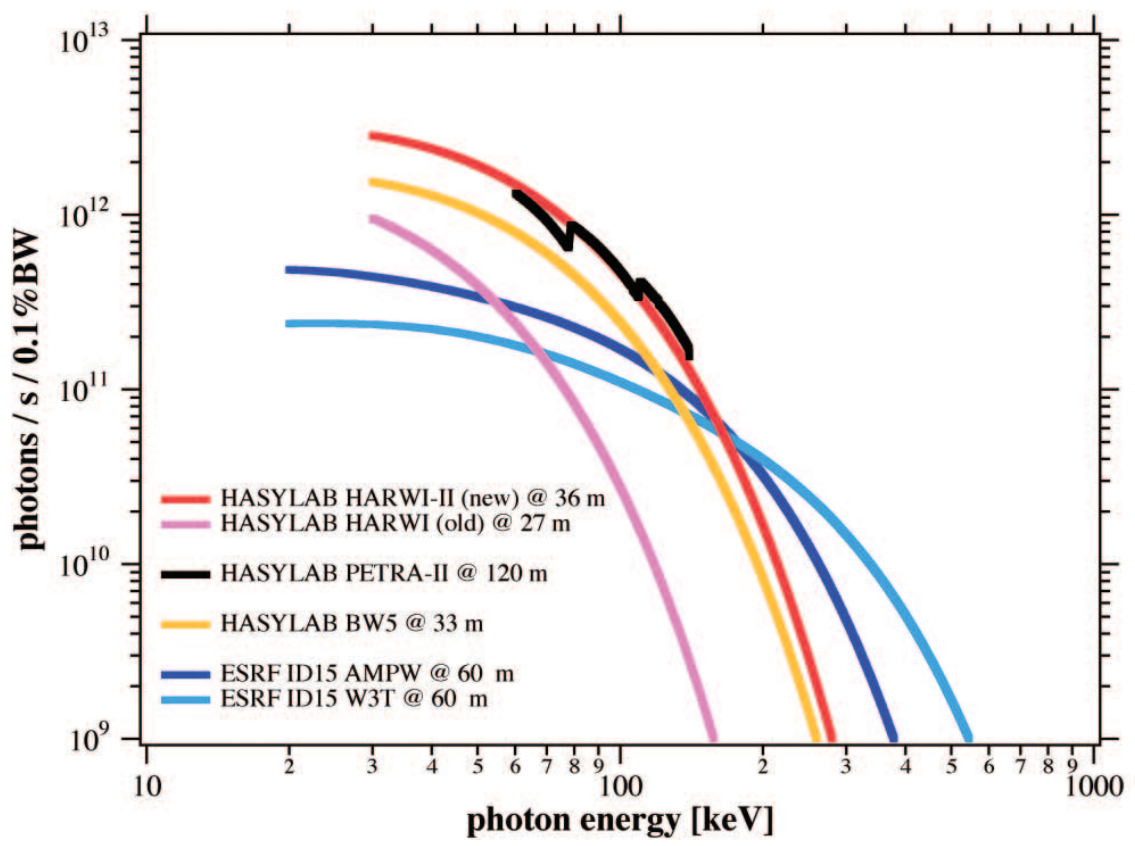

FIGURE 2 Comparison of the photon fluxes of the high-energy beamlines at ESRF and HASYLAB through a $1 \times 1 \mathrm{~mm}^{2}$ pinhole. The medium-energy source HARWI (old) (Hard Röntgen WIggler) is being upgraded by the GKSS Forschungszentrum to HARWI-II (new), which will deliver about twice the flux than HASYLABs existing high-energy beamline BW5 (Beckmann et al., 2003). The short ESRF devices suffer from flux but benefit strongly from a much smaller angular source size. 
and 2 poles, respectively, they are relatively short and suffer from this design on the flux scale. The symmetric devices at HASYLAB are much longer and have about one order of magnitude more poles, which reflects directly in their higher fluxes. Here, even the second-generation devices at DORIS can compete. Their drawback is, however, the huge angular source size, which plays an important role in some applications, like dispersion in strain measurements or for focusing optics.

Before the beam encounters the sample, it has to be conditioned for each individual purpose. Typically a series of metal filters as aluminum or copper of a few millimeter thickness are permanently installed in order to take away the heat load due to lower $\mathrm{X}$-ray energies in the spectrum. Diaphragms and slit systems carefully shape the beam size, for technical, radiation protective and experimental purposes. Either white beam or monochromatic beam can be used according to the individual stations. Of most concern is the monochromator where sets of systems have been developed for Bragg and Laue geometries, focusing and fixed-exit options, high- and low-resolution purposes (Suortti et al., 2001b).

\section{TWO-DIMENSIONAL DIFFRACTION TECHNIQUES}

The most simple experimental technique is the employment of a two-dimensional detector placed behind a sample in transmission mode. High energies have the advantage, that they scatter mainly into the forward direction. The scattering angle $2 \Theta$ is related through Bragg's law to the momentum transfer $Q$ by

$$
\sin (\Theta)=Q / 2 k
$$

where $k$ represents the wavevector, $k=2 \pi / \lambda$, which typically lies in the range of $60 \AA^{-1}$. $Q$ being equal to a reciprocal lattice vector $G$ of up to $10 \AA^{-1}, 2 \Theta$ may reach up to $10^{\circ}$. A good estimate when doing an experiment is the fact that $2 \Theta[\mathrm{deg}] \approx G\left[\AA^{-1}\right]$ at $113 \mathrm{keV}$. A second advantage is the relatively flat Ewald sphere as demonstrated in Fig. 3: Since the energies are about 10 times higher than for conventional X-rays, the curvature of the Ewald sphere is about 10 times smaller. Low-energy Ewald spheres strongly bend away from a plane in reciprocal space, a crystallographic zone, and only a few reciprocal lattice points in some arbitrary directions may lie on or in the vicinity of the sphere. For high energies, we encounter much more the case known from electron diffraction, namely that the sphere smoothly takes off the zone, having reciprocal lattice points from several Brillouin zones in their vicinity.

If, for example, there is some diffuse distribution around these points, one can map a large section of reciprocal space in a single shot. Such an example is given for thermal diffuse scattering on a perfect silicon crystal, in our case along the [111] direction. The bright spots in Fig. 4 originate from the diffuse scattering peaking around the Bragg points and reflect the symmetry of the crystal orientation. Particular branches extend into other crystallographic directions according to the phonon distributions in this material. It is important to mention, that the Bragg peaks themselves are slightly off the Ewald sphere. They would be much sharper and strongly saturate the detector if they were falling onto the sphere. Figures like these are easily obtained on a one-second time scale and can be taken for all crystallographic directions and under 


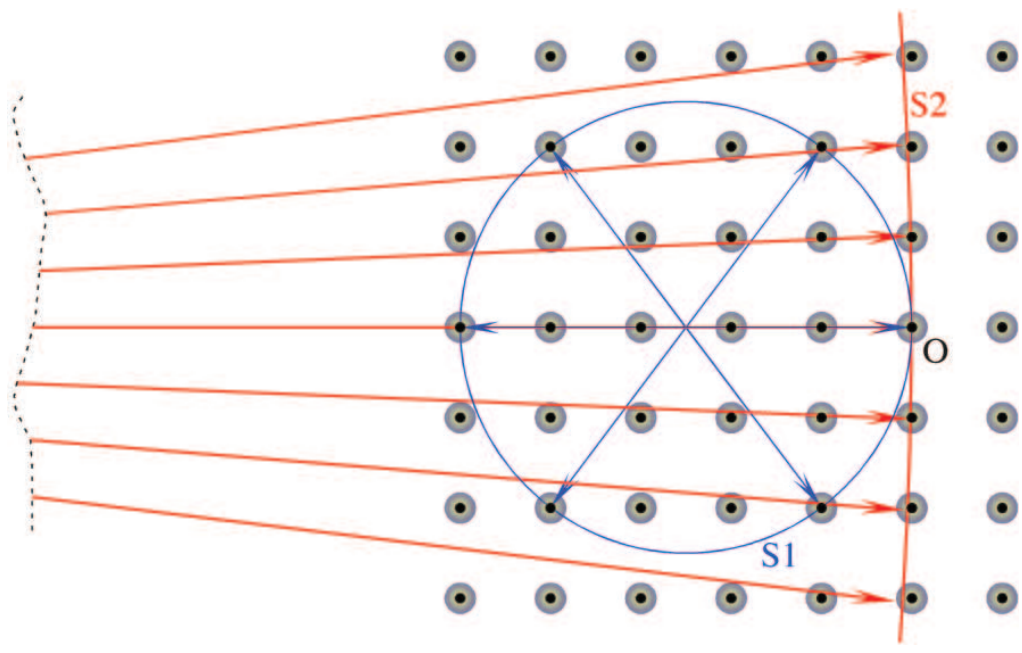

FIGURE 3 Ewald-spheres S1 and S2 for low- and high-energy X-rays, respectively, which differ typically by one order of magnitude in their radius. S1 is strongly curved and only a few reciprocal lattice points may lie in or near to it, the reflected wave vectors going into arbitrary directions. The curvature of S2 is much smaller and the sphere takes off smoothly a plane in reciprocal space. Diffraction goes mainly into the forward direction and diffuse halos around the Bragg peaks can be mapped in a single shot over several Brillouin zones.

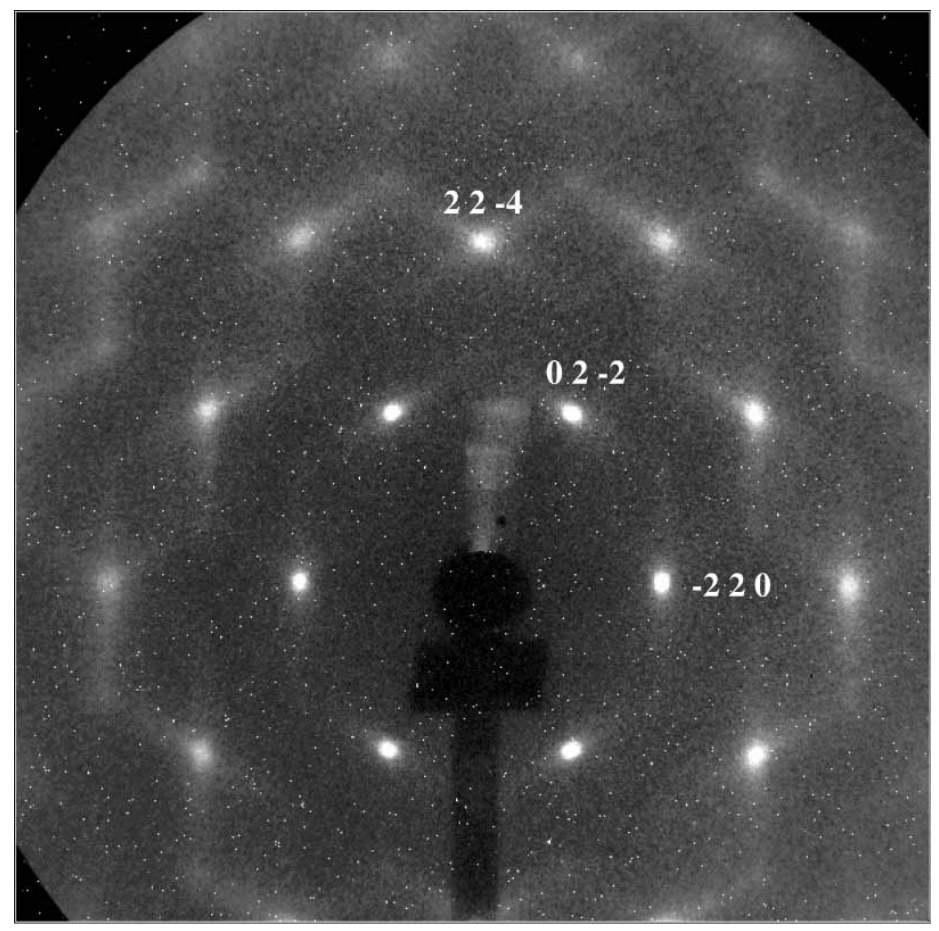

FIGURE 4 Thermal diffuse scattering of silicon taken in the 111 direction with an image intensifier and a CCD camera. The bright spots are centered close to the Bragg peaks which are not directly registered in this setup and show the symmetry of the crystal lattice. Some diffuse branches extend into other crystallographic directions according to the phonon density distribution of the crystal. The direct X-ray background has not been shielded for the current setup and hits the CCD chip resulting in the scatter of saturated pixels 


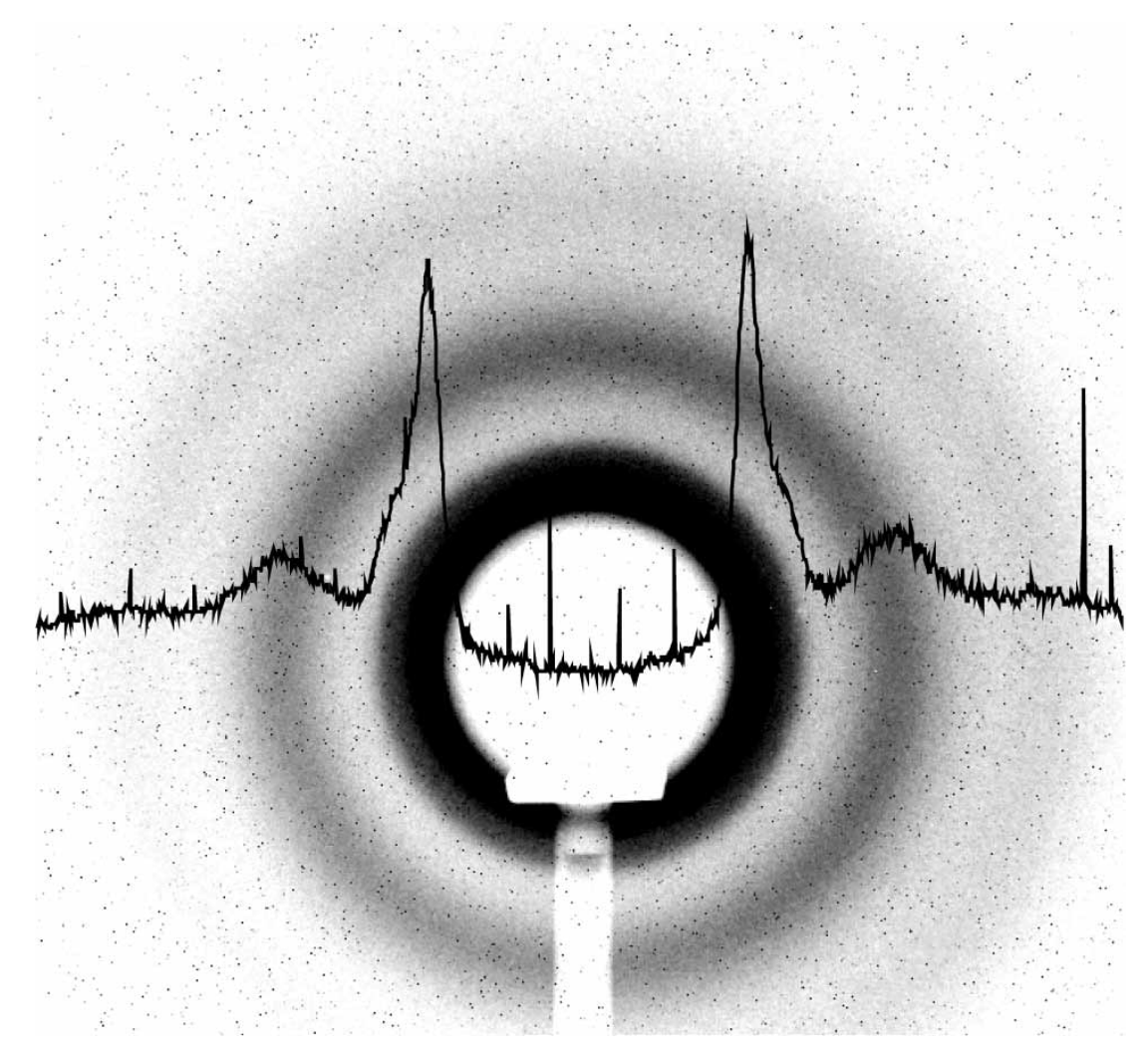

FIGURE 5 Scattering from liquid InGa, registered with an image intensifier tube coupled to a CCD camera. The black curve is a horizontal cut through the center. The data can be acquired within a second of time and demonstrates the potential for in-situ observations on liquid and amorphous materials. The very sharp peaks are artefacts due to X-ray photons from the background hitting the CCD chip directly and deposing a large charge. They can be shielded properly or corrected by a numerical image treatment.

influences of external parameters. Diffuse scattering as well plays an important role to study phase transitions and this technique has been employed successfully on systems like the spin glass $\mathrm{Cu}_{83} \mathrm{Mn}_{17}$ (Reichert et al., 2001).

Studies on liquids and amorphous samples necessitate the registration of the scattering function $S(Q)$ up to high-momentum transfers of $30 \AA^{-1}$ or even more, where highenergy X-ray diffraction becomes necessary to access this $Q$-range at all (Neuefeind and Liss, 1996). Different scanning techniques have been employed for careful measurements, but the simple setup with a two-dimensional detector is of great benefit with regard to the speed of data acquisition. Figure 5 shows a diffraction pattern of the liquid eutectics InGa with the radial intensity distribution superimposed. The rings stem from the distributions of neighbors in the liquid. The fast data acquisition with a CCD camera allows for in-situ studies as undercooling and nucleation processes for crystallization as studied by several groups and other applications in the field of liquids and amorphous materials (Notthoff et al., 1999; Tomberli et al., 2001; Holland-Moritz et al., 2002; Marques et al., 2002).

Polycrystalline materials benefit from the registration of entire Debye-Scherrer rings with a two-dimensional detector. A typical setup is given using an image-plate 


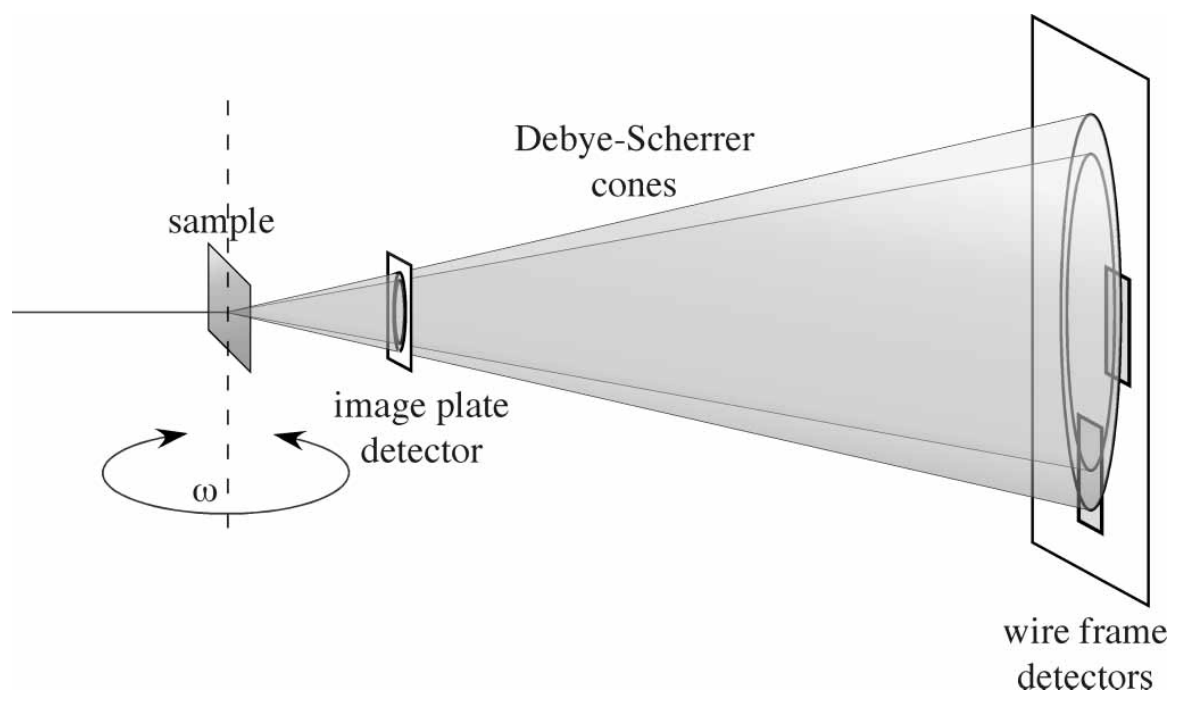

FIGURE 6 Setup for the registration of Debye-Scherrer rings of a polycrystalline sample in order to measure strain or texture. An image plate detector can be used in 1-2 $\mathrm{m}$ distance to register the whole pattern. Alternatively, ring sections can be recorded more accurately by putting larger area detectors further away. Such a configuration has been established by using wire-frame gas detectors at the PETRA-II beamline at HASYLAB (Kampmann et al., 2001).

detector as shown in Fig. 6. Here, both, the eccentricity of a ring and the azimuthal intensity distribution along the ring can be used for strain and texture measurements, respectively. Since these applications are overlapping with other techniques, they are treated in an individual chapter.

\section{TEXTURE AND STRESS ANALYSIS}

The quantitative determination of stresses in work pieces is a most important issue in order to understand the deformation behavior of materials and to simulate manufacturing processes as well as to predict the lifetime of a structural component. Because polycrystalline materials consist of single crystallites (grains, second phases, precipitates, etc.) which can exhibit a significant mechanical anisotropy, most of the mechanical properties and strengths depend strongly on the texture of the material. Textures are the distribution functions of orientations of the crystallites. Preferred orientations are obtained, for example, by the production process, such as rolling or extrusion. Thus, "texture engineering" is used to influence and control the mechanical and physical properties of a material. On the other way round, textures can tell about the production history of a material. Finally, it should be noted that geologists learn about deformation processes in the earth crust by measuring the textures of rocks (Günther et al., 2002).

Textures are easily obtained by the registration of entire Debye-Scherrer rings of several reflections simultaneously and evaluating the intensity distribution $I$ around the ring as the first independent angle $\gamma$ for the pole figure (Wcislak et al., 2001,2003). A second, independent angle $\omega$ is obtained by rotation of the sample around an axis perpendicular to the incident beam. The algorithm for the translation 
of the angles $(\theta, \gamma, \omega)$ into a pole figure $(\alpha, \beta)$ can be found in the literature (Bunge and Klein, 1996). Basically, the Debye-Scherrer ring at $\omega=0$ describes a circle with $\alpha=90^{\circ}-\theta$ and $\beta=\gamma$, which can be expressed in Cartesian coordinates $(x, y, z)$ for a point on the unit sphere. Further a rotation matrix $M(\omega)$ has to be multiplied to this vector in order to get the final position in the pole figure. A stereographic or alternatively a parallel projection then relates to the final representation $(x, y, I)$. The Debye-Scherrer rings for a rolled sample of $\gamma$-TiAl as registered at $87 \mathrm{keV}$ on the PETRA-II beamline are shown in Fig. 7 for the two extreme $\omega$-rotations. The sheet material is $\mathrm{Ti}-46.5 \mathrm{Al}-4(\mathrm{Nb}, \mathrm{Mn}, \mathrm{Cr}, \mathrm{Si})-0.5 \mathrm{~B}$ (at $\%$ ) a second-generation $\gamma$-TiAl-based alloy called $\gamma$-TAB (Kestler et al., 1999). The microstructure is "near gamma," that means fine-grained and equi-axed with a very low content of second phase $\alpha-\mathrm{Ti}_{3} \mathrm{Al}$. The structure is ordered $\left(\mathrm{L}_{0}\right)$ and tetragonal with a $c / a$-relation of about 1.02 . The 001 ring is a superlattice reflection and shows, in Fig. 7(a), intensities only in transverse direction $(t)$ and does not appear, in Fig. 7(b), in which the transverse direction is parallel to the beam. The 002 and 200 reflections generate a double ring due to the tetragonality. The inner 002 ring occurs, in Fig. 7(a), only in transverse direction, whereas the outer 200 ring is visible only in rolling direction $(r)$. In Fig. 7(b) the 002 ring is without intensities, but the 200 ring is observed in rolling direction $(r)$ as well as in normal direction $(n)$ of the sheet. The observed intensity distribution on the rings are caused by a very strong cube texture component with an alignment of the $c$-axis [001] of the tetragonal unit cells in transverse direction and the $a$-axis in the rolling and normal

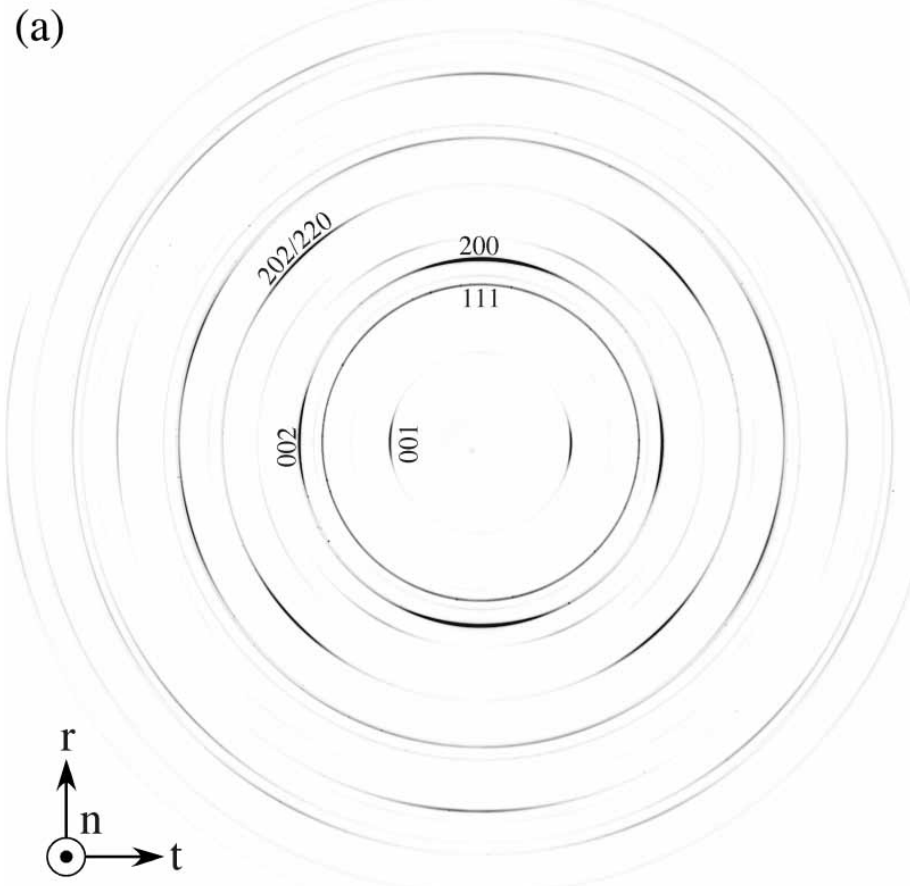

FIGURE 7 Debye-Scherrer rings of a highly textured $\gamma$-TiAl sample for $\omega=0^{\circ}$ (a) and $\omega=90^{\circ}$ (b). Strong differences can be seen in the intensity distribution of each ring, which are coupled through the crystalline symmetry to each other. 
(b)

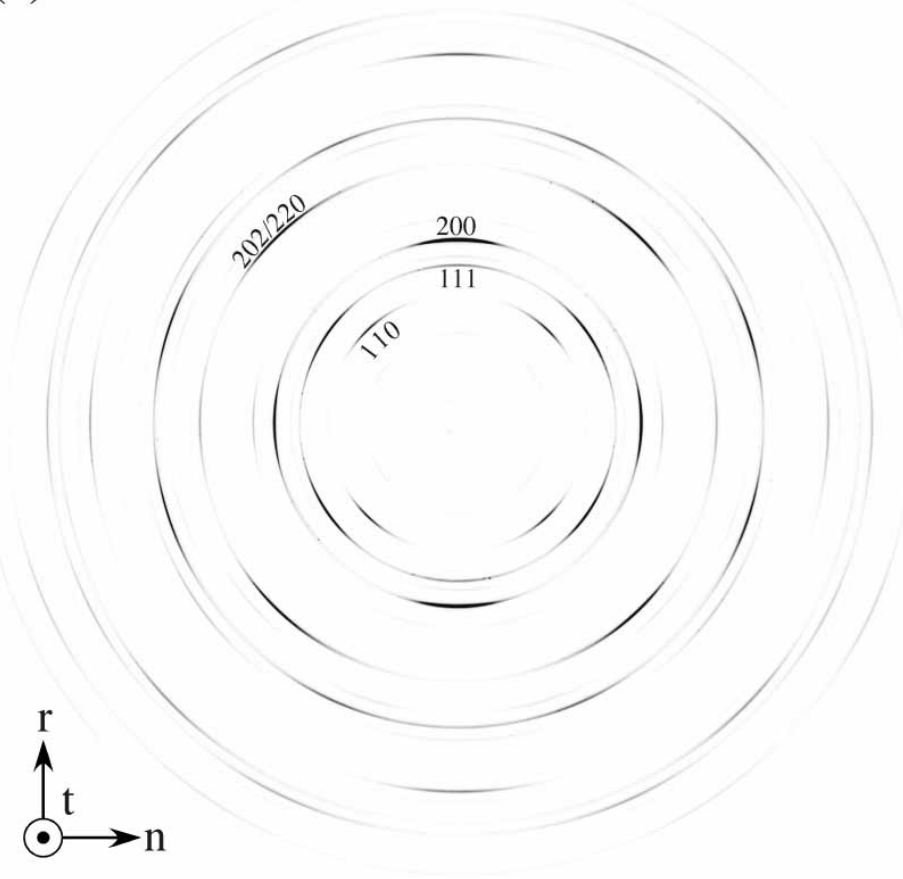

FIGURE 7 Continued.

direction. This texture is the result of recrystallization during the reheating between two rolling passes and causes a strong anisotropy of the mechanical behavior (Bartels et al., 2002, 2003; Schillinger et al., 2002).

Pole figures are evaluated for some particular reflections and represented in Fig. 8 in form of a parallel projection. They show the high texture of the material. The 001 directions are concentrated around the transverse direction. The 111 pole figure shows that the structure of the TiAl is near to fcc and we see the reflection of the four planes of the 111 tetrahedron oriented accordingly to the cube component of the texture. The 220/ 202 pole figure shows all possible 6 reflections of the unit cell in cube orientation. The pole figures confirm that the "modified cube texture" with the aligned $c$-axis is the sole texture component in the $\gamma$-TiAl alloy sheet.

The most accurate two-dimensional detectors in terms of spatial linearity and dynamic range are image plate detectors. Although sophisticated online-models can be achieved commercially, they have the drawback to be slow in readout time lasting 2-3 min. ${ }^{1}$ The exposure time, often less than $1 \mathrm{~s}$, is in no relation to that! In order to overcome this disadvantage, moving detector techniques have been developed, which register only part of a Debye-Scherrer ring through a fixed aperture. Then the detector is displaced like a streak camera synchronously to the sample movement (Wcislak et al., 2003). Another attempt to surround this problem is to employ CCD cameras with

\footnotetext{
${ }^{1}$ Model MAR 345 by MAR, Evanston, IL, USA; http://www.marresearch.com/
} 

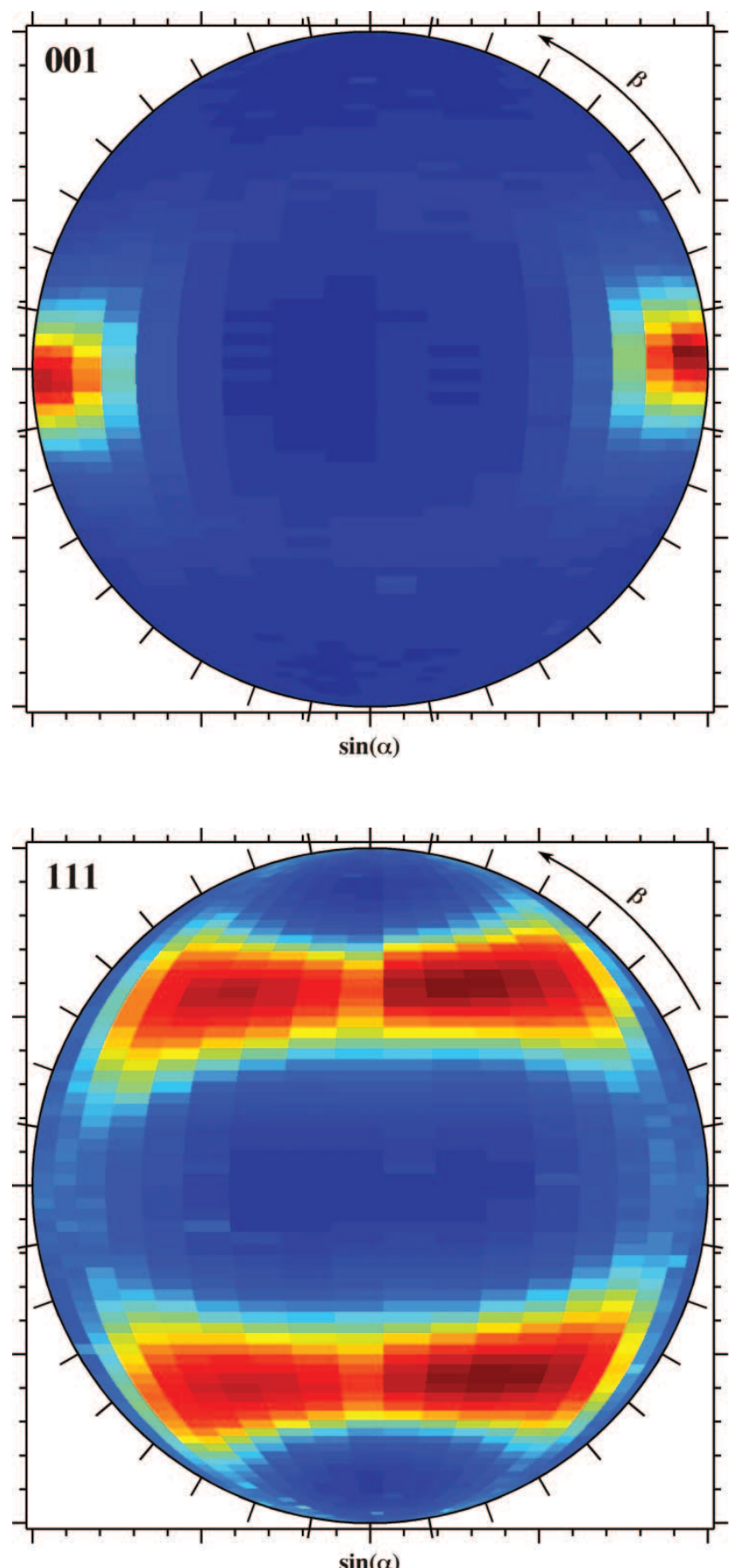

FIGURE 8 Parallel projections of the pole figures with the polar angle $\alpha$ and the azimuthal angle $\beta$ of the 001,111 and 220/202 reflections of a highly textured $\gamma$-TiAl sample. The figures have been constructed from the Debye-Scherrer rings of 10 different $\omega$-orientations between $0^{\circ}$ and $90^{\circ}$. The $c$-axis of the slightly hexagonal material lies along the direction transverse to the rolling direction which points upwards in the figures. 


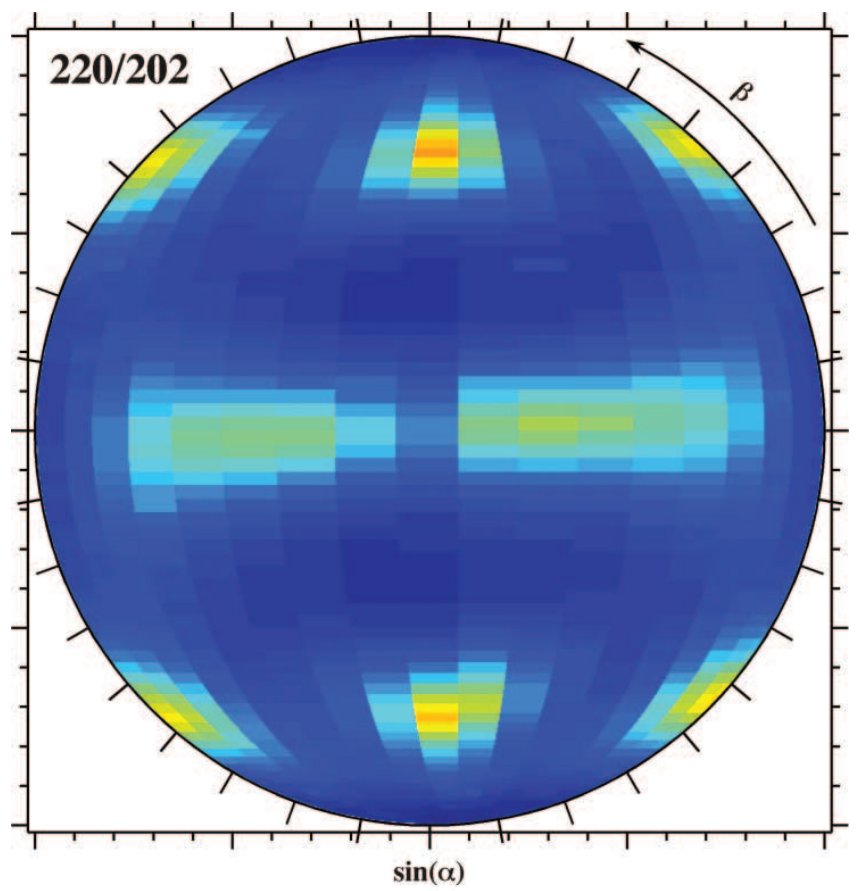

FIGURE 8 Continued.

some visual light or 'image intensifier' optics as pursued by groups at the ESRF (Terry et al., 2002).

Residual stresses occur in workpieces or structural components after deformation or/and heat-treatments. For example, bending of a metal bar can lead to elastic and plastic deformation. After loosing the bending force, the plastic component holds the bar further bent within an equilibrium curvature, while potential elastic forces at the inner and outer radius become compressive and tensile. They remain as residual stresses and either inhibit or enhance, respectively, the formation of cracks. Their technological aspects are huge and can be a nuisance or a benefit.

Stresses cannot be measured directly. They are evaluated from longitudinal strain measurements $\varepsilon_{k k}=\Delta d_{k k} / d$ which can be obtained by neutron- or X-ray diffraction in the spatial dimension $k$. Then, the corresponding stresses $\sigma_{i i}$ are calculated with the elastic stiffness tensor $C_{i j k l}$ (which has to be taken from the literature or from a dedicated experiment) according to Hooke's law:

$$
\sigma_{i j}=C_{i j k l} \varepsilon_{k l}
$$

The radiation is sensitive to the lattice parameter $d$ through Bragg's law (2) which reads with $Q=G=2 \pi / d$

$$
\lambda=2 d \sin (\Theta) .
$$

The logarithmic derivative, i.e. the total derivative of (4) divided by (4) itself and solved for the strain $\varepsilon=\Delta d / d$ relates to 


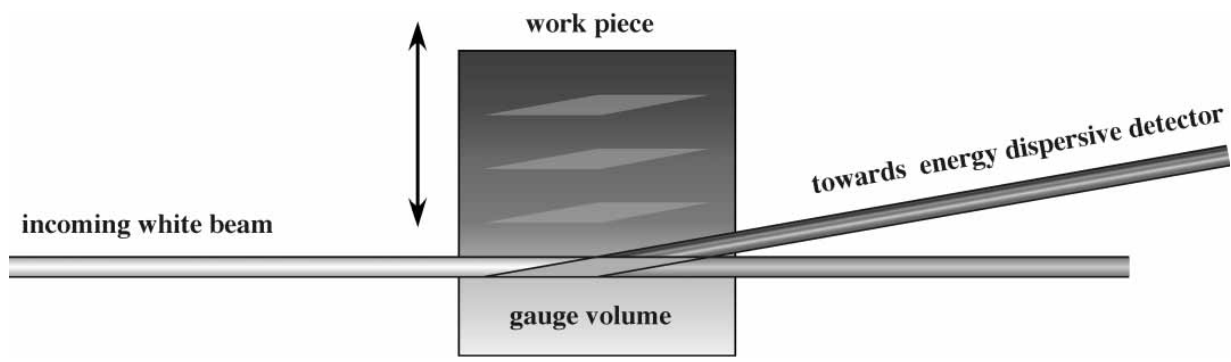

FIGURE 9 Energy-dispersive setup. The incoming and the diffracted beams are laterally limited by a system of slits or collimators, such that only the overlapping area of sight defines a gauge volume. Due to the small scattering angle, its length is typically one order of magnitude larger than its width. The workpiece can be scanned through the gauge volume in order to register strain gradients as a function of position and orientation.

$$
\Delta d / d=\Delta \lambda / \lambda-\cot (\Theta) \Delta \Theta,
$$

i.e. strain can be measured through two complementary methods, either wavelength or angular dispersive. Since the wavelength is correlated to the energy of the radiation, the former method is also called energy-dispersive.

Energy-dispersive techniques were the first to be established for high-energy beamlines (Brusch, 1998). The setup is sketched in Fig. 9, where an incident, white beam is collimated by a tiny slit of $50-100 \mu \mathrm{m}$ onto the polycrystalline sample. A secondary slit system behind the sample defines the scattered beam towards the energy-dispersive germanium detector. It fixes the scattering angle, which is typically chosen around $6^{\circ}$ at the dedicated diffractometer of ID15A. It also defines a gauge volume by the set of all sample volume elements illuminated by the incident beam and seen through the slit system from the detector. The shape of this gauge volume is given by the lateral beam dimension and the scattering angle, resulting in some $50-100 \mu \mathrm{m}$ in width and up to $1 \mathrm{~mm}$ in length. Crystallites from an ideal powder reflect according to Bragg's law into the detector and select their corresponding energy according to the crystallographic reflection. A typical pattern obtained from a multi-phase A1Si25Cu4Mg1 alloy $(\mathrm{wt} \%)$ is given in Fig. 10. This case demonstrates well the individual fingerprints of the constituting phases which can be considered separately. A full description and evaluation of these data is given in Pyzalla et al. (2001). Under load, the crystal lattice is deformed and the strain yields in a slight shift of the corresponding energy according to $\Delta d / d=-\Delta E / E$. Now, the sample can be probed in different volumes in order to obtain a spatial strain distribution and/or under external load such as the force from a tensile rig or to investigate the influence of temperature on a layered system of coatings which exhibit different thermal expansion coefficients. The data acquisition at one point lasts typically $15 \mathrm{~min}$ but may depend strongly on the material. Accuracies down to $10^{-4}$ may be achieved by appropriate care and fitting. The application of this method ranges from the in-situ examination of composite materials (Pyzalla et al., 2001), thermal barrier coating systems (Reimers et al., 1999) and functionally graded materials (Reimers et al., 1998; Broda et al., 1999). A drawback of this method is the need for the exact knowledge of the average center of the scattering volume. Particularly, when a sample is scanned through its position and the phase probed for enters or exits the gauge volume without filling it up completely results in peak shifts due to the shift of the average scattering angle (Brusch, 1998). In addition, the described 


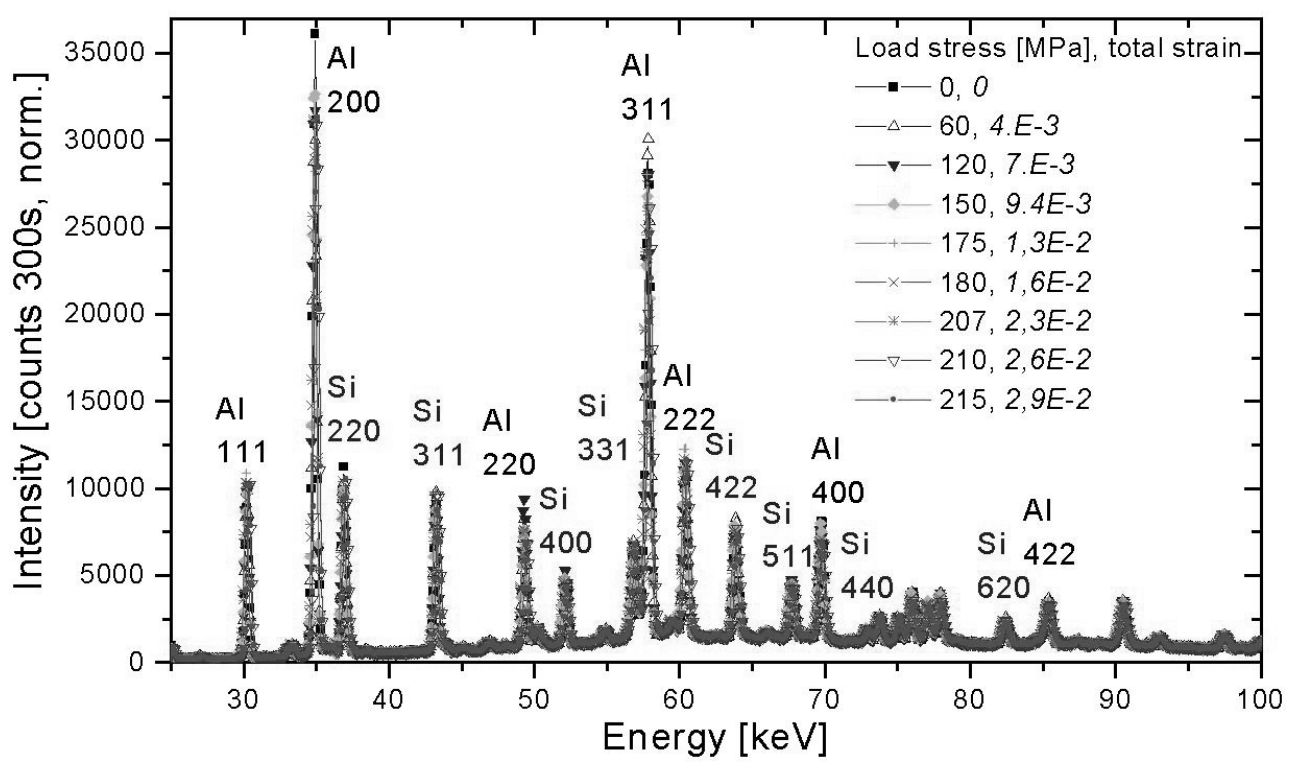

FIGURE 10 Energy-dispersive diffraction pattern of a multi-phase AlSi25Cu4Mg1 alloy under different mechanical loads. It is clear, that the constituting phases can be evaluated separately. The effect of the strain is very small on this full scale and can be barely seen by eye. Careful fitting routines, however, evaluate it to an error of $10^{-4}$. It is clear, that the silicon and aluminum parts can be evaluated separately due to their individual fingerprints in the pattern. From (Pyzalla et al., 2001).

method works only for grains small enough to have a smooth distribution of intensity over the whole gauge volume. Again, a larger grain in the scattering condition, off-centered the gauge volume results in a shift in the mean scattering angle and thus gives a systematic error. If grains become too large, they may not scatter into the detector position at all and the method cannot be used any more.

A more flexible technique is the registration of Debye-Scherrer rings with a monochromatic incident beam. The setup is qualitatively similar to the one for acquiring texture measurements, albeit more care has to be taken to account for a high strain resolution. The strain is derived from (5), best with $\Delta \lambda / \lambda=0$. It is expressed in the diameter of a Debye-Scherrer ring with respect to a starting condition. Thermal expansion, for example, increases the lattice spacings with increasing temperature, which through (4) relates to a shrinkage of the rings. On externally applied, uniaxial stress, however, the lattice parameter expands along the force direction while it shrinks with Poisson's ratio in the perpendicular direction. This relates to shrinkage and expansion of the radius of the Debye-Scherrer rings in the respective azimuthal orientations. The rings become ellipses and two components of the strain tensor can be measured simultaneously. Let $D$ be the distance between the sample and the detector, then the radius $R$ of a Debye-Scherrer ring reads

$$
R=D \tan (2 \Theta)
$$

If the Bragg angle is small as it is the case in high-energy diffraction, we can develop the sine and the tangent function in (2) and (6) to $Q \approx G / 2 k$ and $R \approx 2 D Q$ resulting in

$$
\varepsilon=\Delta d / d=-(\Delta D / D+\Delta R / R) .
$$


The relative change in the diameter is directly the negative value of the strain component and is a quantity easy to measure. However, care has to be taken to keep the resolution and errors in $\Delta R$ small: First, the detector resolution is not smaller than $100 \mu \mathrm{m}$ and secondly a final size of the incident beam reflects directly in the width of the Debye-Scherrer rings. The last quantity has to be minimized to the order of the resolution of the detector or a bit smaller. Let us assume the realistic values of an intrinsic width of the Debye-Scherrer rings of $150 \mu \mathrm{m}$ on the image plate in $1.5 \mathrm{~m}$ distance and a ring radius of $150 \mathrm{~mm}$. Then the full width at half maximum in $\varepsilon$ results in $10^{-3}$ and cautious fitting routines determine the strain to $10^{-4}$, the accuracy we aim to measure with this method. Equation (7), however, expresses further, that the distance between the sample and the detector must not vary well beyond $150 \mu \mathrm{m}$. Indeed, this condition is not so easy to hold. Experiments are often done under in-situ environments, for example in a tensile rig or in a furnace, which makes it difficult to prevent a drift of the sample position. In practice, a calibration powder which sticks at the sample surface is measured simultaneously, which, however, may lead to other problems, especially during experiments conducted at high temperatures. In addition, the sample thickness should not broaden the full width at half maximum such that thicknesses above $1 \mathrm{~mm}$ should be avoided. One way out of these constraints is to place the detector 5-10 times farther away from the sample. If we aim for a similar resolution in $\varepsilon$, all the numbers scale up with the distance increase. Especially the influence of the position drift of the sample falls one order of magnitude less into account and the registration of a calibration powder is not necessary. A great distance, however, means huge Debye-Scherrer rings and no detectors have been set up yet covering the whole area of the rings. Instead it has been demonstrated to register only part of the rings and to determine their curvature by a fitting method (Böhm et al., 2003). In particular two detectors can be used in order to still acquire two dimensions of the strain field. However, in a greater distance the requests regarding spatial resolution of the detectors is relaxed and position sensitive gas detectors as developed by $\mathrm{DENEX}^{2}$ can be employed (Kampmann et al., 2001; Marmotti et al., 2002). Those detectors are not isotropic and the position resolving wires are oriented radially to the Debye-Scherrer ring in order to receive a resolution of about $1 \mathrm{~mm}$. As a feasibility experiment, uniaxial tensile tests were conducted on intermetallic $\gamma$-TiAl based alloy sheet material. The data, obtained with the image plate detector with $D=1150 \mathrm{~mm}$ and a photon energy of $100 \mathrm{keV}$ at BW5 are shown in Fig. 11. The same experiment has been repeated at PETRA-II employing two wire-frame detectors with $D \approx 7 \mathrm{~m}$. The size of the detectors was $300 \times 300 \mathrm{~mm}^{2}$ and the experimental setup is sketched in Fig. 6. The raw data of the two experiments are compared in Fig. 12. One important difference is the fact, that many more rings are resolved at the PETRA-II rather than the BW5 setup. The investigated $\gamma$-TiAl based alloy consists of the $\gamma$-TiAl (major phase) and about 10 volume percent of the $\alpha_{2}-\mathrm{Ti}_{3} \mathrm{Al}$ phase. This latter is better distinguished at PETRA-II, which is due to the different beamline parameters, such as a more brilliant source, a more perfect monochromator and a large distance between the sample and the detector. The eccentricity of the rings are evaluated in Fig. 13, which plots the ring diameters of the $\gamma$-TiAl 222 reflection of the sample under applied stress of $470 \mathrm{MPa}$ as a function

\footnotetext{
${ }^{2}$ DENEX GmbH, Lüneburg, Germany; http://www.denex-gmbh.de/
} 


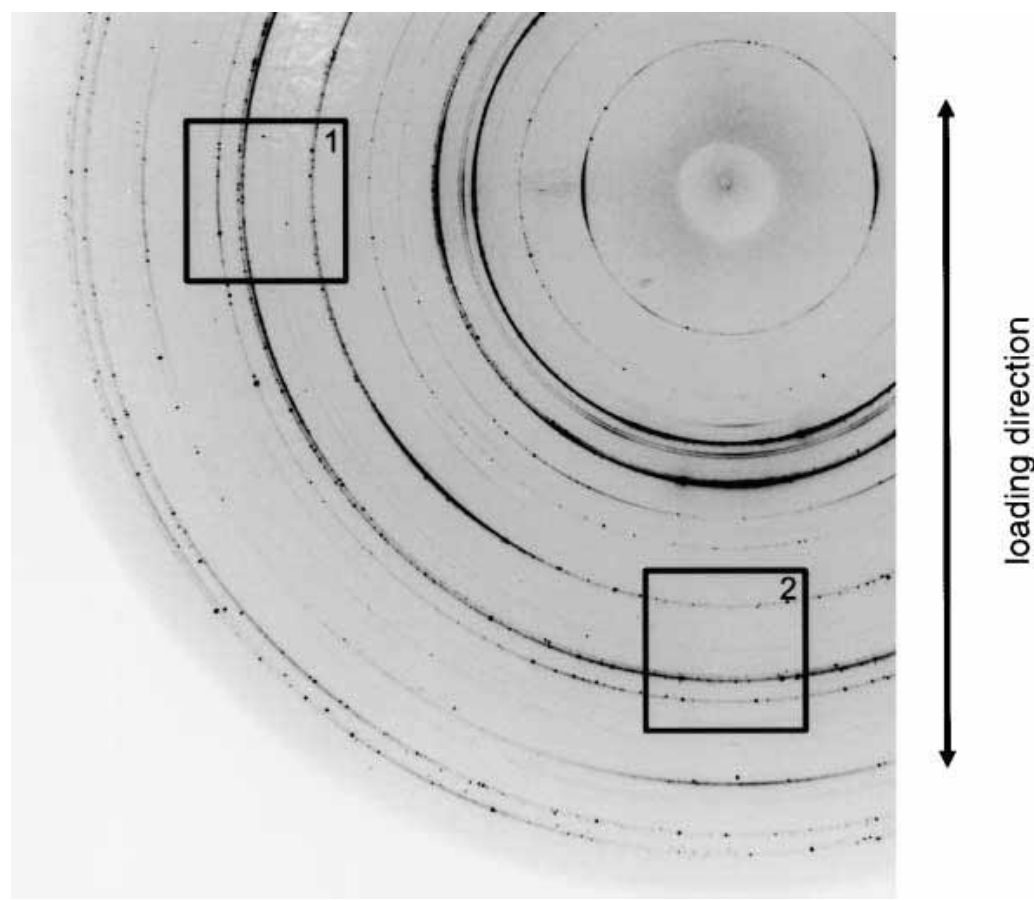

FIGURE 11 Part of a typical diffraction pattern of the $\gamma$-TiAl-based alloy, recorded by the image plate detector at beamline BW5. A close-up of area "1" is shown in Fig. 12(a). From Böhm et al. (2003).

of the azimuthal angle $\phi$. The diameters are normalized to the diameter obtained from the unloaded sample and show the eccentricity with the short and long axes along and perpendicular to the load direction with $\sin ^{2} \phi=1$ and $\sin ^{2} \phi=0$, respectively. The scatter of the points around the mean straight line is a direct measure for the statistical error and the salient result of this comparison is the better accuracy of the PETRA-II experiment by at least a factor of 5. A full evaluation of this work has been published in an individual paper (Böhm et al., 2003).

\section{RESOLUTION WITH TWO-DIMENSIONAL DIFFRACTION}

It has been shown that better accuracy can be achieved for strain analysis with the Debye-Scherrer method if a larger distance between the sample and the detectors is used (Böhm et al., 2003). This is one important, but not the only issue which plays a role when defining an experimental setup or a beamline. Of similar importance are the angular source size, which makes up a new-generation synchrotron source and the quality of the optics employed. The angular source size is the angle under which the source is seen from a slit just in front of the sample position. This divergence determines, how large the beam spreads onto the detector and must be minimized for strain measurements. For texture, single crystal or even small angle scattering applications has to be guaranteed, that adjacent reflections do not fall into a larger divergence and overlap. Further, it is desirable that the source size is similar in vertical and horizontal dimensions. However, the necessary use of a monochromator 


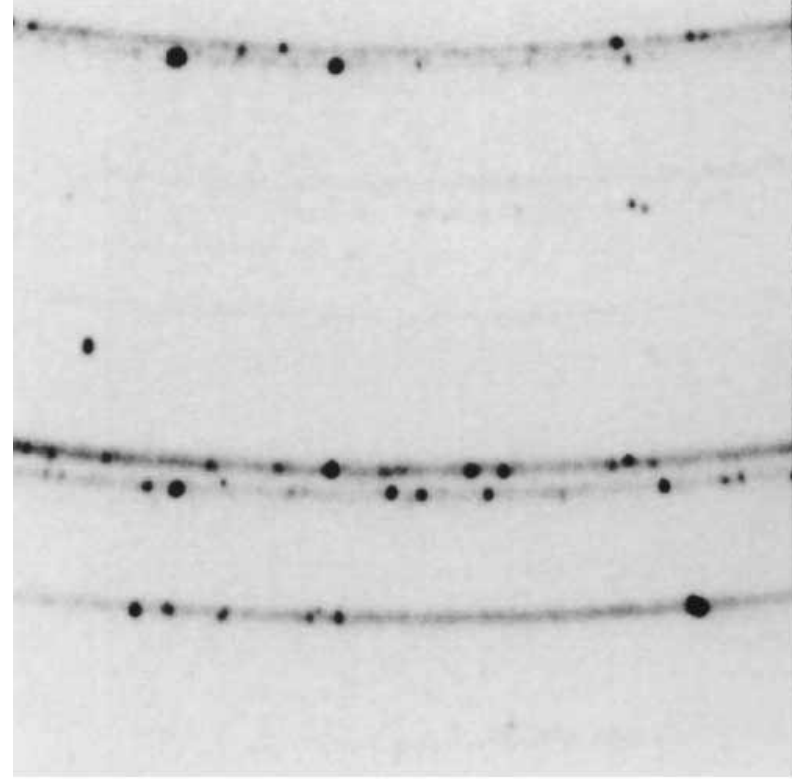

(a)

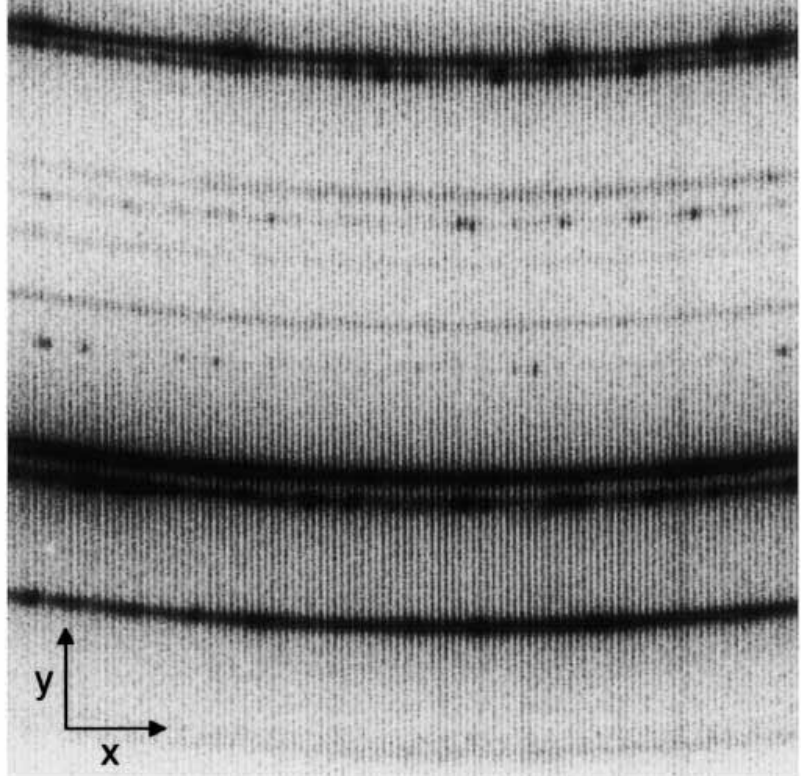

$\underset{\gamma-220}{\gamma-202}$

$\gamma-003 / \alpha_{2}-302$

$\gamma-221$

$\alpha_{2}-203$

$\gamma-301$

$\alpha_{2}-400$

$\gamma-113 / \alpha_{2}-222$

$\gamma-311 / \alpha_{2}-401$

$\gamma-222 / \alpha_{2}-004$

$\alpha_{2}-402$

(b)

FIGURE 12 Corresponding diffraction patterns (a) and (b) registered by the image plate detector at beamline BW5 (area "1" of Fig. 11) and by one of the two wireframe area detectors at PETRA2, respectively. The reflections are indexed on the right for the phases $\gamma-\mathrm{TiAl}_{\text {and }} \alpha_{2}-\mathrm{Ti}_{3} \mathrm{Al}$. From $\mathrm{Böhm}$ et al. (2003). 

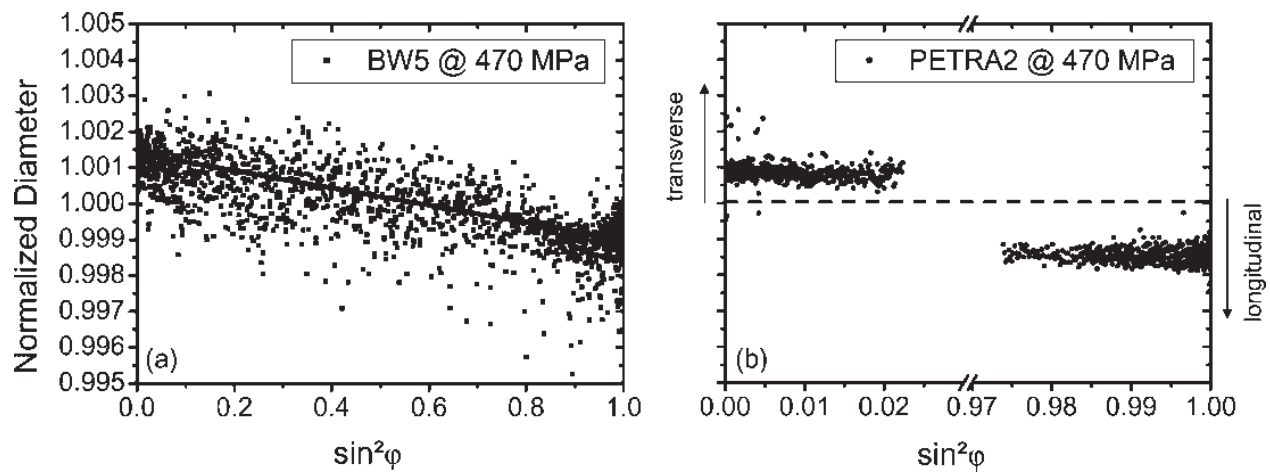

FIGURE 13 (a) Example of the strain evaluation procedure based on the full diffraction rings recorded at BW5. The diameter of the $\gamma$-TiAl 222 diffraction ring is plotted against $\sin ^{2} \phi$, where $\phi$ is the azimuthal angle. The diameters are normalized to the average ring diameter in the stress-free starting condition. Due to the lattice strains caused by the applied stress of $470 \mathrm{MPa}$, the diffraction ring is distorted elliptically. The two main axes of the ellipse are obtained by fitting a straight line to the data and extrapolating to $\sin ^{2} \phi=1$ (longitudinal direction) and $\sin ^{2} \phi=0$ (transverse direction). (b) The same procedure can be applied to the radii of the ring segments recorded at PETRAII. The range of $\sin ^{2} \phi$ is restricted to values close to 0 and 1 . It is apparent that the data in (b) exhibit considerably less scatter than those in (a). From Böhm et al. (2003).

is a dispersive optical element with highest anisotropy. Here, we have to distinguish between the particular nondispersive and the dispersive setups, as sketched in Fig. 14 . Imagine a setup of two crystals, first with identical lattice spacing, the monochromator and the sample as sketched in Fig. 14(a) where a divergent, white incident beam hits the first crystal with Bragg angles $\theta_{1}, \theta_{2}, \theta_{3}$ for the different rays to the reflecting lattice planes. Since each ray has a different Bragg angle, different wavelengths $\lambda_{1}, \lambda_{2}, \lambda_{3}$ are selected, which is called dispersion. Now these rays impinge onto the second crystal which has to be rotated into Bragg condition and, remaining in a common scattering plane, two positions either rotating the scattered beam into the same sense $(++)$ or the inverse sense $(+-)$ are possible. In the former, Bragg condition for the first ray can be fulfilled by impinging $\lambda_{1}$ by $\theta_{1}$. Then, however, the other rays do not impinge with their appropriate angles to the net planes, thus only $\lambda_{1}$ is reflected, the others transmitted. By rotating further the second crystal, $\lambda_{2}$ and $\lambda_{3}$ come into Bragg condition successively while reflection fails for the first ray. With this fully dispersive geometry one mainly probes for the beam divergence. Considering the $(+-)$ geometry it turns out that the lattice planes of the second crystal have to be parallel to the first in order to get $\lambda_{1}$ under $\theta_{1}$ into reflection. Then, however, one has $Z$-geometry and mathematically all $\lambda_{i}$ enter with their corresponding $\theta_{i}$ being reflected all at a given rotation angle. Further all rays loose the Bragg condition commonly when the crystal is rotated such that the angular width of the reflection curve is minimal. The beam divergence does not play any role and this mode is called the nondispersive one. The setup is sensitive to monochromator and sample parameters like lattice spacing or mosaicity only. Since each polycrystalline material is constituted of small single crystallites, these results from single crystal diffractometry also play a role in the Debye-Scherrer setup described above, which, however, have to be generalized for more parameters of freedom: First, the nondispersive condition is only valid for a point on the detector, the non-dispersive point $Z$, which falls into the scattering plane of the monochromator and corresponds to the scattering angle $-2 \Theta_{\text {mono }}$ in $(+-)$ geometry. The diffraction conditions may differ in two orthogonal ways from this point, namely by an azimuthal 
(a)

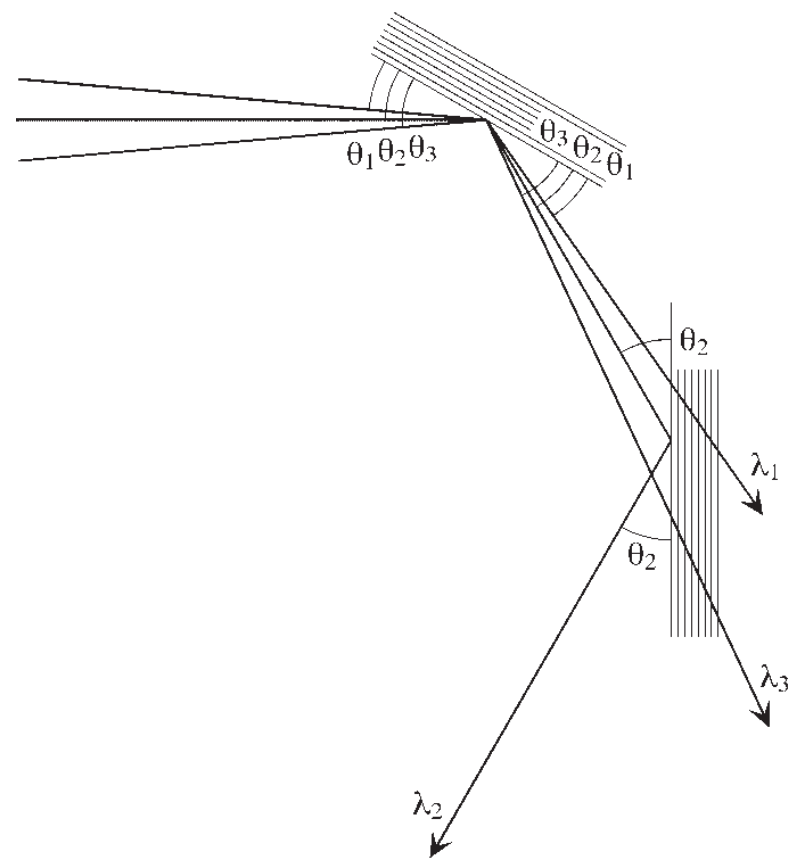

(b)

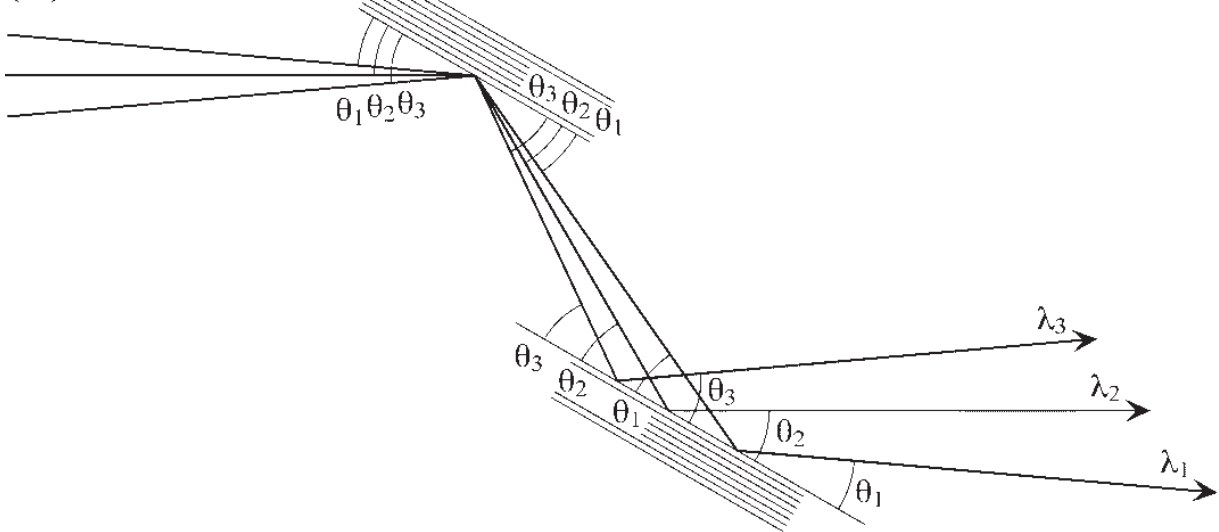

FIGURE 14 Dispersive (a) and nondispersive (b) two-crystal arrangements, $(++)$ and $(+-)$ geometry, respectively, within a common scattering plane. A divergent, white beam impinges under different Bragg angles onto the first crystal selecting different wavelengths for each ray. While all rays are reflected simultaneously with their corresponding Bragg angles in the $(+-)$ setup, the second crystal has to be rotated to fulfill Bragg's law successively in the $(++)$ arrangement. (a) is sensitive to the beam parameters while (b) measures the crystal qualities upon scanning.

rotation going along a Debye-Scherrer ring until one reaches the $(++)$ geometry, or by a variation in the lattice spacing or scattering vector, going radially. The farther one goes away from the nondispersive point, the more dispersion plays a role. It can be shown, that the streaks all point toward the nondispersive point $Z$ (100) in Fig. 15. 


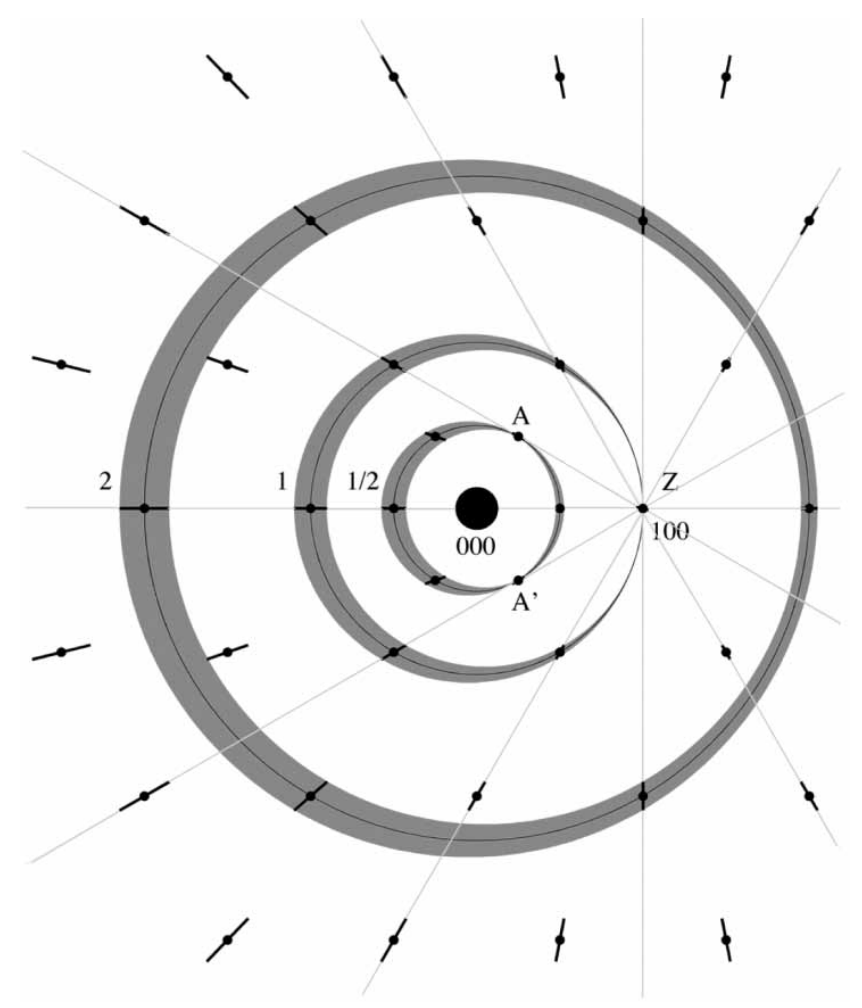

FIGURE 15 Dispersion of the diffracting system monochromator-sample. The figure sketches a diffraction pattern as it should be observed with a two-dimensional detector placed behind the sample. The spots can be either single crystal reflections registered in a rotating crystal technique or reflections from individual crystallites in a polycrystalline sample. Usually a beam stop covers the direct transmitted beam 000 , the origin of reciprocal space. The reciprocal lattice point 100 denotes the nondispersive point, to which all resolution streaks of the other points point to. The length of the resolution streak vanishes at this point and increases linearly with the distance. This effect influences as well the width of Debye-Scherrer rings as drawn here with radii $1 / 2,1$ and 2 times the length of the scattering vector of the monochromator. For the inner rings, points $\mathrm{A}$ and $\mathrm{A}^{\prime}$ exist where the resolution streak is aligned tangentially to the ring and the thickness of the rings vanishes.

Reversing the arguments, such a diffraction pattern reveals the employed monochromator reflection and orientation.

The previous thoughts hold for both, single crystal diffraction and the characterization of Debye-Scherrer rings. Experimental data are given in Fig. 16. The behavior of the width of the rings along their azimuthal coordinates can be simulated by drawing two off-centric rings delimiting the intensity which include the gray areas in Fig. 15. The width of the rings vanishes, of course, at the nondispersive point and becomes maximal on the opposite side (ring 1). For $G>G_{0}$, dispersion always delivers a nonvanishing ring width (ring 2). In the opposite case $G<G_{0}$, however, these two off-centric rings intersect in the positions $A$ and $A^{\prime}$ where the resolution elements become tangential to the rings (ring 1/2). Large angles around $90^{\circ}$ between $A$ and $A^{\prime}$ can be achieved by an appropriate design of the monochromator and this might be interesting in setting up an experiment to measure with high accuracy the two independent strain components 
along these directions, which has never been done so far. A more quantitative analysis of this resolution effect will be published elsewhere.

\section{TRIPLE-AXES DIFFRACTOMETRY}

Single crystal studies with high energies play an important role in physics and materials science. Especially high resolution in reciprocal space is obtained with a triple-axis diffractometer and detailed descriptions, strategies for reciprocal space mapping and resolution are given in dedicated papers (Neumann, 1991; Neumann et al., 1994; Rütt et al., 1995; Bouchard et al., 1998; Liss et al., 1998b; Rütt et al., 2001). Investigations of lattice distortions in perfect (Liss et al., 1997a; 1998a) and imperfect materials (Bouchard et al., 1993; Liß, 1994; Magerl et al., 1995; Helmers, 1996), studies of macroscopic thick or industry relevant samples (Royer and Bastie, 1997; Royer et al.,

(a)

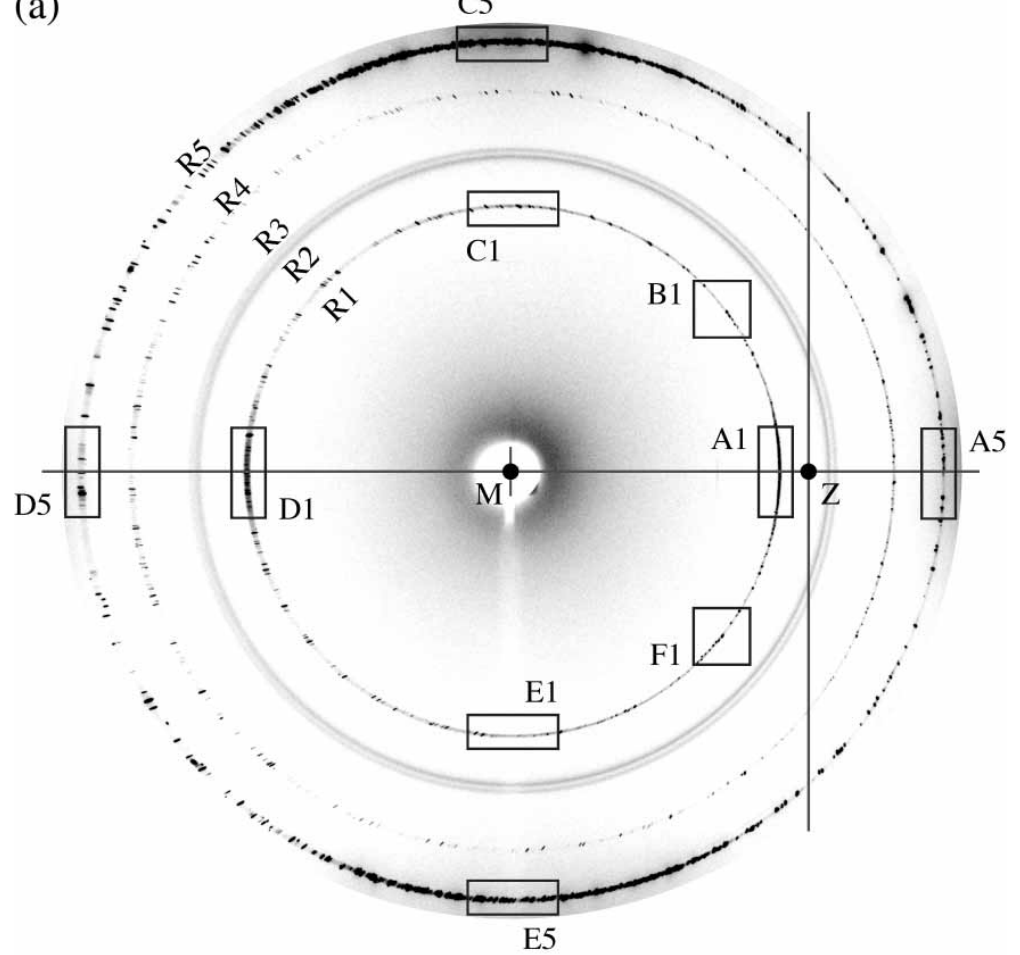

FIGURE 16 (a) Experimental Debye-Scherrer rings R1-R5 obtained at $90 \mathrm{keV}$ on TiAl (R1, R4, R5) and a further window materials (R2, R3) centered around $M$. The rings demonstrate dispersion with the nondispersive point $Z$ as reconstructed from the data. They are fine and well distinguished close to $Z$ while they broaden further away from this point. Some of the rings, R2 and R3 even begin to overlap on the opposite side of the nondispersive point. The streaks stemming from individual crystallites point all towards $Z$. The boxes A1-F1 and A5-F5 are expanded in Fig. 16(b) and (c), respectively.

(b) Expansion of part of the outer Debye-Scherrer ring R5 from Fig. 16(a). The dispersion streaks as mapped by the reflections of individual crystallites are exactly pointing to the nondispersive point $Z$ as indicated by the arrows. Note, that the ring is narrowest in the $(+-)$ geometry (A5) and broadest in (++) (D5).

(c) Expansion of the inner Debye-Scherrer ring R1 from Fig. 16(a). Again, the dispersion streaks are well aligned towards the non-dispersive point $\mathrm{Z}$ as shown by the arrows. In B1 and F1, they are aligned tangentially towards the rings which is the position, where the rings become narrowest. 


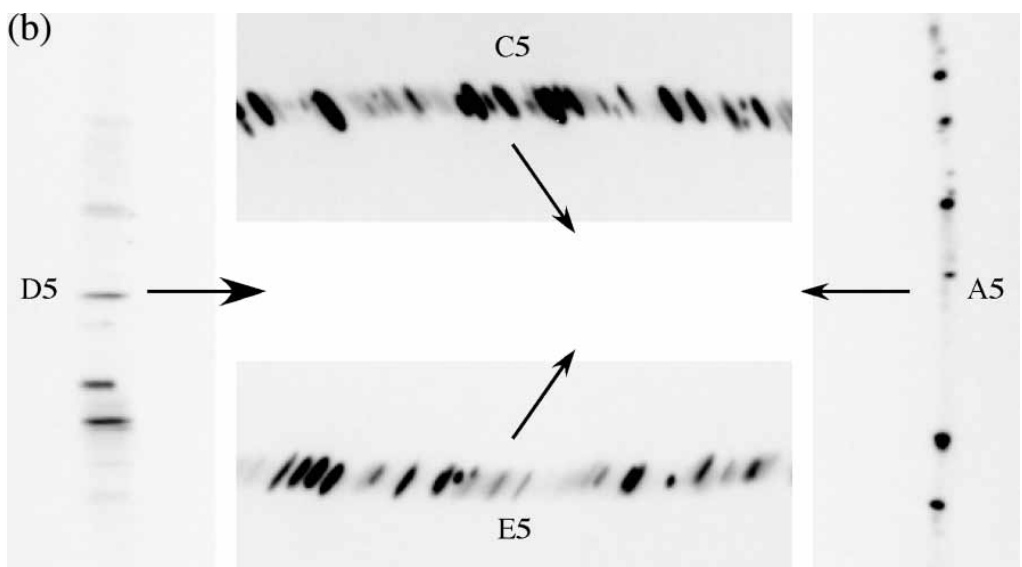

(c)
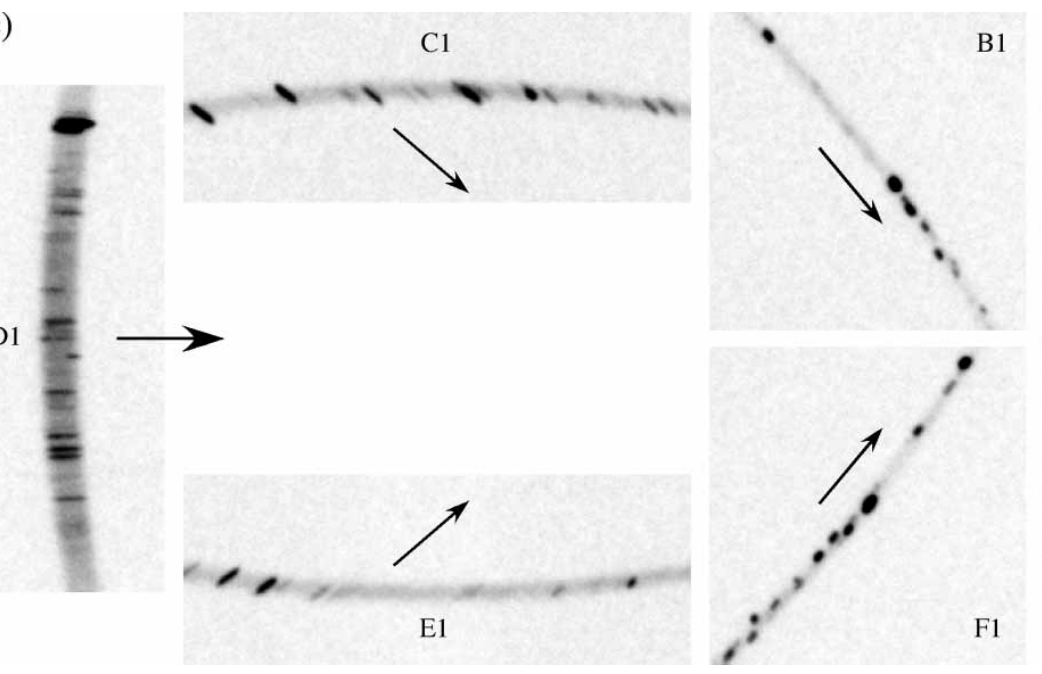

B1

FIGURE 16 Continued.

1997a,b; Goorsky et al., 1999), structural phase transitions in the bulk of a material (Neumann et al., 1995; Poulsen et al., 1996; Wilkins et al., 2000; Lippmann and Schneider, 2000a,b; Arzel et al., 2002,2003; Hatton et al., 2003) and their kinetics (Liss et al., 2003), diffuse diffraction from 'relaxor' ferroelectrics as discussed by (Zhukov et al., 1995), nonresonant magnetic diffraction (Lippert et al., 1994; Strempfer et al., 1996) and anomalous thermal expansion near a magnetic phase transition (Chatterji et al., 1998) are only a few of the applications which have successfully used this technique.

A typical layout of a triple-axes diffractometer is given in Fig. 17 which consists of three individual axes each holding a crystal, namely the monochromator, the sample and the analyzer. The essential tactics in reciprocal space is sketched in Fig. 18. The monochromator, defines an incident wavevector $\boldsymbol{k}_{i}$, which in the Ewald construction, points to the origin 0 of reciprocal space whereas the analyzer accurately probes for 


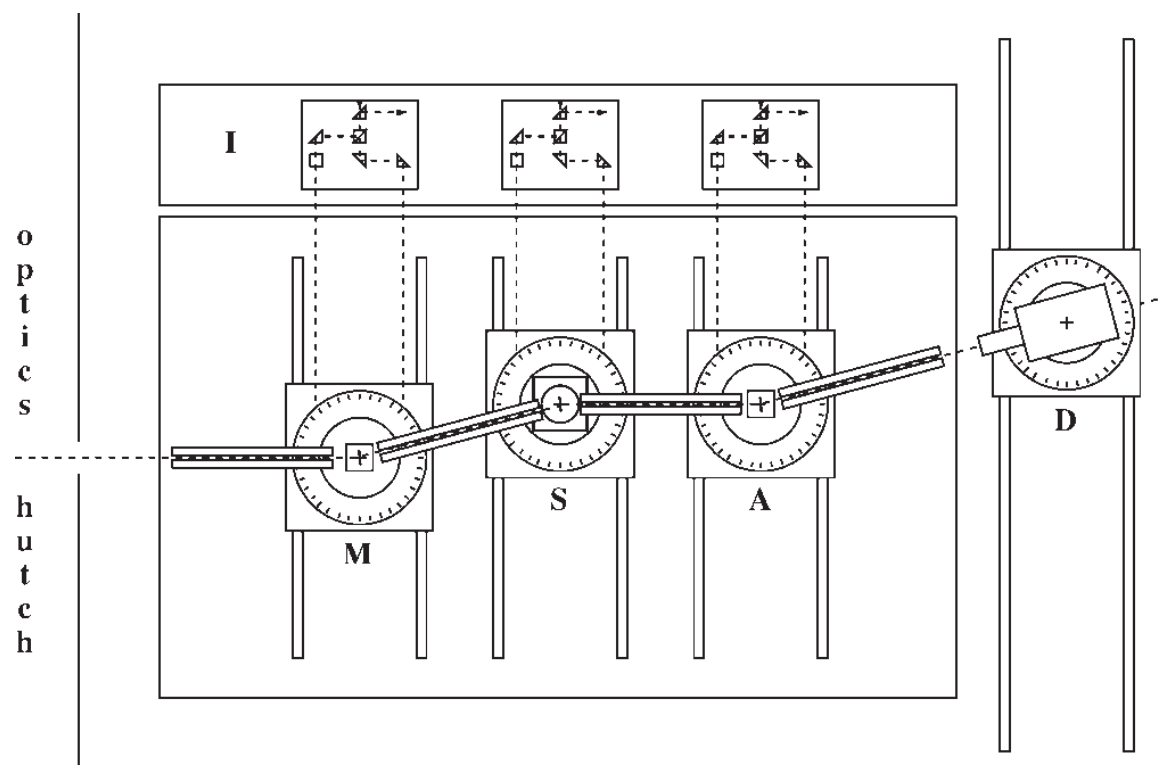

FIGURE 17 Schematic layout of the triple crystal diffractometer at ID15A. A pre-monochromatized or white beam impinges from the optics hutch to the left, the sources being about $65 \mathrm{~m}$ away. The beam path is fed through the monochromator $\mathbf{M}$, sample $\mathbf{S}$, and analyzer $\mathbf{A}$ positions in order to meet the detector $\mathbf{D}$. Collimators in-between reduce the solid angles and thus the background. All components are mounted onto translation stages and are run computer-controlled into the correct position. Interferometric encoders I can be used for ultimate angular resolution beyond 0.01 arc sec. Either perfect or oxygen-precipitated monochromators $\mathbf{M}$ and analyzers A can be used to compromise between resolution and intensity. From Liss et al. (1998b).

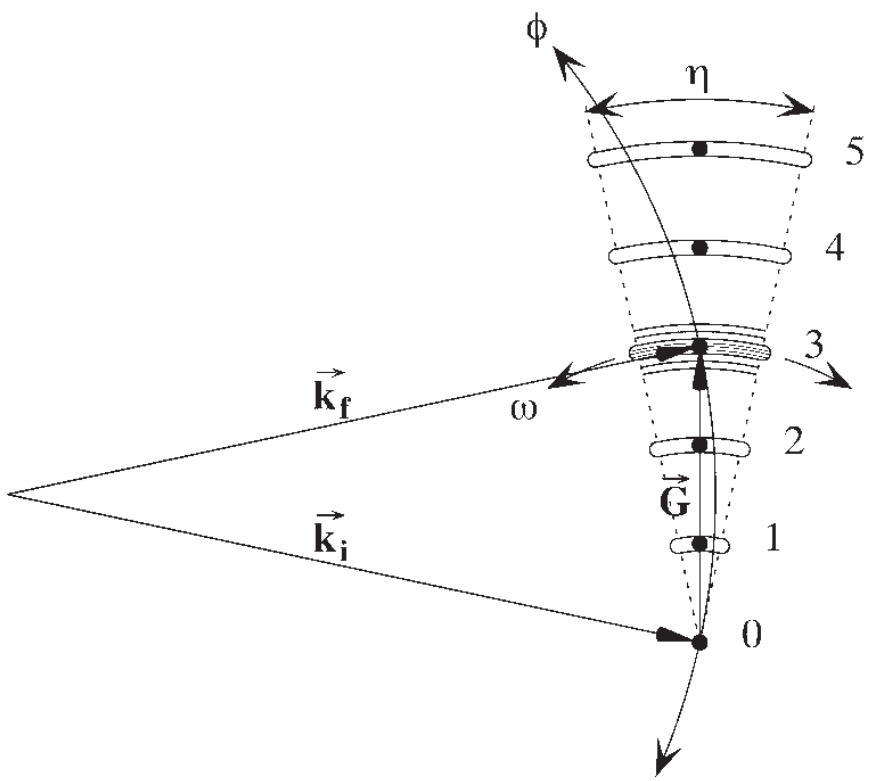

FIGURE 18 Strategy for reciprocal space mapping with a triple axes diffractometer. The monochromator defines well the incident wave vector $\boldsymbol{k}_{i}$, the analyzer accurately fixes $\boldsymbol{k}_{f}$, both lying on the Ewald sphere and probing for a scattering vector $\boldsymbol{G}$ in the reciprocal lattice distribution of the sample. Transverse scans are obtained by the $\omega$-rotation of the sample while the longitudinal direction, inclined by the small Bragg angle $\theta$, is obtained by a $\phi$-rotation of the analyzer. 
a diffracted wave vector $\boldsymbol{k}_{f}$. The Bragg condition is fulfilled, if a scattering vector $\boldsymbol{G}$ of the sample lies on the Ewald-sphere such that

$$
\boldsymbol{G}=\boldsymbol{k}_{f}-\boldsymbol{k}_{i}
$$

holds. Here, the analyzer acts as an extremely narrow collimator accepting rays in the very narrow angular range of its natural line width. This triple-crystal mode has to be distinguished from the two-crystal mode omitting the analyzer crystal and thus integrating over a larger angular range in $\phi$.

Usually, $\boldsymbol{G}$ is not a point in reciprocal space, but a distribution, which can be mapped out in two dimensions, i.e. longitudinal, $\Delta G_{\|}$and transverse, $\Delta G_{\perp}$ to the scattering vector. The scanning parameters are the sample and analyzer rotation angles $\omega$ and $\phi$, respectively and can be transformed to the local, Cartesian coordinates in the vicinity of $\boldsymbol{G}$ by

$$
\left[\begin{array}{c}
\frac{\Delta G_{\|}}{G} \\
\frac{\Delta G_{\perp}}{G}
\end{array}\right]=\left[\begin{array}{cc}
\frac{1}{2(\tan (\theta)} & 0 \\
\frac{1}{2} & 1
\end{array}\right] \cdot\left[\begin{array}{c}
\Delta \phi \\
\Delta \omega
\end{array}\right]
$$

(Liss et al., 1998b). Thus, $\omega$ probes for the mosaic spread of the sample while $\phi$ seeks mainly for the longitudinal distribution and i.e. is most sensitive to the lattice parameter. Resolutions of $10^{-6}$ and $10^{-7}$ can be reached for the longitudinal and transverse directions, respectively.

The triple-axes diffractometer enjoys dedicated applications since over more than a decade and most of the instrument functions have been described in the literature cited above, such that at this point we limit the presentation to a very few, selected examples.

Applications in materials science are interesting for a quality mapping of single crystals, grown for the many different applications and techniques. Rocking curves in two-crystal mode can be taken locally as a function of the position of a larger sample (Goorsky et al., 1999). Figure 19 shows the local full width at half maximum (FWHM) of an $11 \mathrm{~mm}$ thick, Verneuil grown crystal of $\mathrm{SrTiO}_{3}$ as obtained from the company CRYSTAL GmbH. ${ }^{3}$ It is mostly narrow, thus the crystal is almost perfect in the center of its volume, from where growth has started, while mosaicity strongly spreads in the outer regions. Triple crystal investigations are necessary in order to distinguish different phases and compositions such as the phases of nickel-base superalloys interesting for the engineering of turbine blades of aircraft engines (Royer and Bastie, 1997; Royer et al., 1997a,b) or the epitaxial growth of $\mathrm{Si} / \mathrm{Ge}$ alloys in the semiconductor industry. Figure 20 gives an example with seven layers of $\mathrm{Si}_{1-x} \mathrm{Ge}_{x}$ with seven different concentrations $x$ which have been grown epitaxially by a rapid CVD method onto a substrate of perfect silicon (Madar et al., 1992; Liß, 1994; Magerl et al., 1994). One can distinguish the very narrow substrate peak at $\Delta G_{\|} / G=0$ from the seven epitaxial peaks extending to $\Delta G_{\|} / G=-15.5 \times 10^{-3}$ due to the different lattice parameters of the individual concentrations. They reveal increasing transverse widths related to a

\footnotetext{
${ }^{3}$ Crystal Gmbh, Berlin, Germany; http://www.crystal-gmbh.com
} 


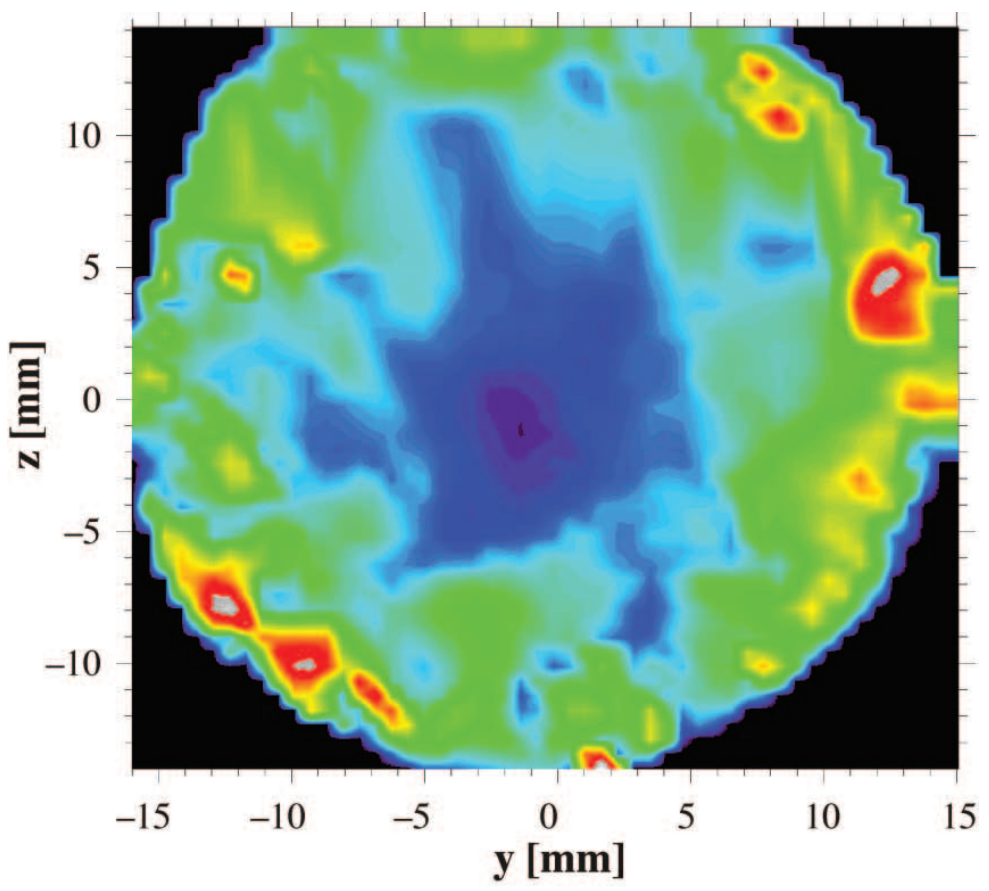

\section{local FWHM [degree]}

0

0.08

0.16

FIGURE 19 Spatial distribution of the full width of half maximum (FWHM) of rocking-curves taken on a $11 \mathrm{~mm}$ thick $\mathrm{SrTiO}_{3}$ crystal with $200 \mathrm{keV}$ radiation. The crystal was Verneuil grown, starting in the center. The figure demonstrates, how the crystal quality detoriates from a nearly perfect lattice alignment in the center to a large mosaic spread in the outer regions.

continuous introduction of lattice faults in order to relax the crystallographic lattice mismatch.

The quality of ultrasonic fields, which have been excited into a volume of a perfect silicon crystal can be mapped and diffraction effects can be studied in detail. Thus low-frequency sound oscillations up to the $10 \mathrm{MHz}$ range represent an anisotropic strain field which oscillates as a function of space and time. X-rays diffracted in Laue geometry integrate over several ultrasonic wave periods along [111] and probe for the strain distribution as demonstrated in Fig. 21 for the $\mathrm{Si}_{5} 31$ reflection (Liss et al., 1997b). An unexcited crystal shows the natural line width of 0.2 arc sec and $50 \%$ of reflectivity in the Laue case as obtained by the dynamical theory of diffraction (Rauch and Petrascheck, 1976, 1978). An ultrasonic excitation induces a gradient with maximal strain proportional to the total width of the reflection curve. It depends on the voltage applied to the exciting transducer. The evaluation of the shape of the rocking curve reveals the quantitative strain distribution in the crystal (Liss et al., 1998). The doubling in intensity is related to the loss of the Pendellösung effect in a gradient crystal (Liß, 1994). Since the ultrasonic field oscillates as a function of time, stroboscopic measurements can be acquired with the time resolution of 100 ps of the synchrotron. Figure 


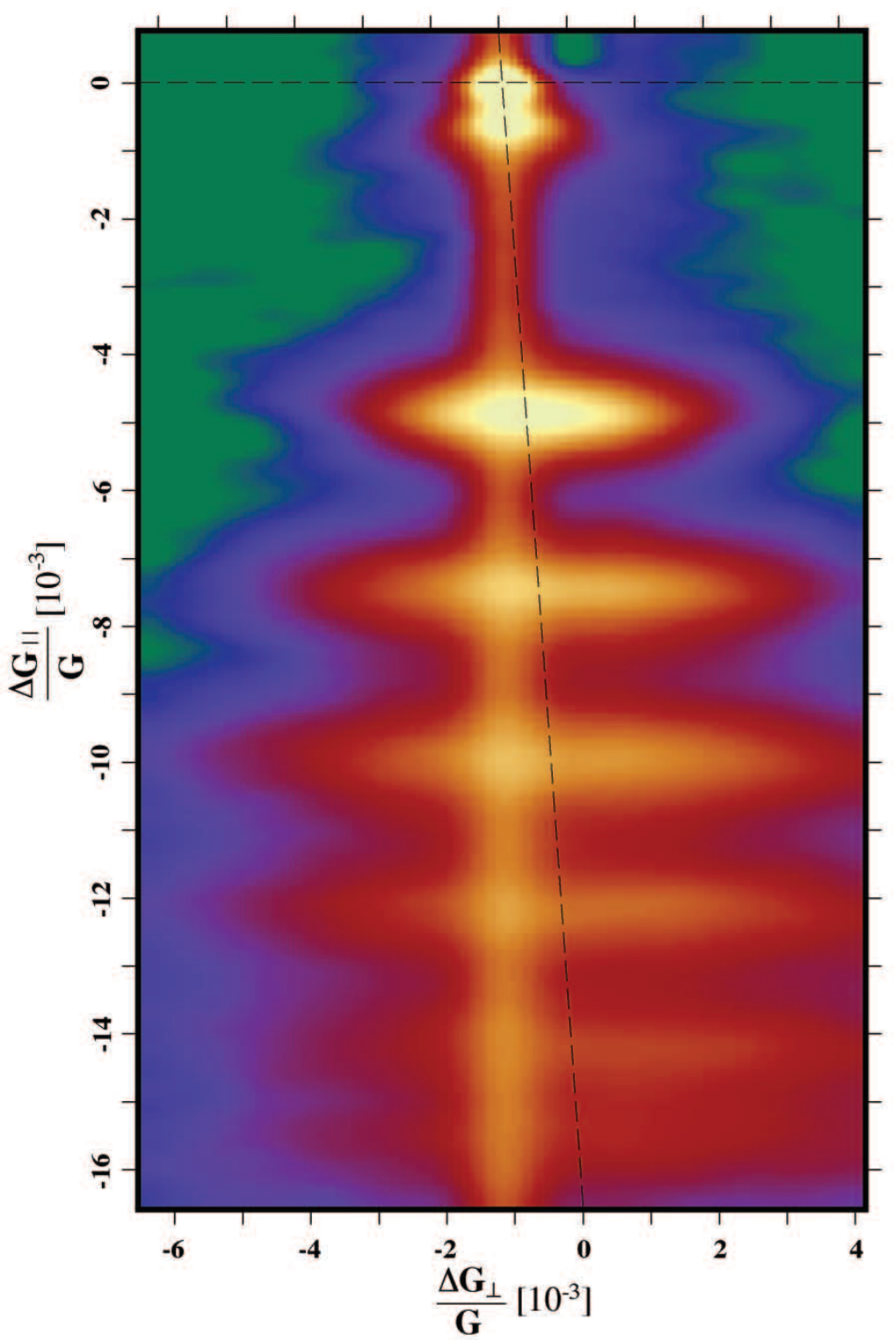

FIGURE 20 Diffraction pattern on rapid-CVD grown $\mathrm{Si}_{1-x} \mathrm{Ge}_{x}$ layers on a silicon substrate with seven steps of concentration $x$. Each composition with individual germanium content has its own lattice parameter which is distinguished by its longitudinal deviation $\Delta G_{\|} / G$ in reciprocal space, while the mosaic spread as a result from lattice tilts as created by misfit dislocations or stacking faults appear in the transverse direction $\Delta G_{\perp} / G$. The crystal grew starting from the silicon substrate at $\Delta G / G=0$ with stepwise increase of germanium, showing less crystal quality for higher concentrations. From Liß (1994).

22 demonstrates a harmonic oscillation with temporal nodes and antinodes in the strain distribution. A full evaluation is given in a dedicated paper (Liss et al., 1998a). For high ultrasonic frequencies above $100 \mathrm{MHz}$, the diffraction pattern becomes qualitatively different: The ultrasonic wave forms a superlattice to the crystalline structure which can be resolved using the ultimate resolution of the instrument, here with a $\mathrm{Si}_{7} 51$ 


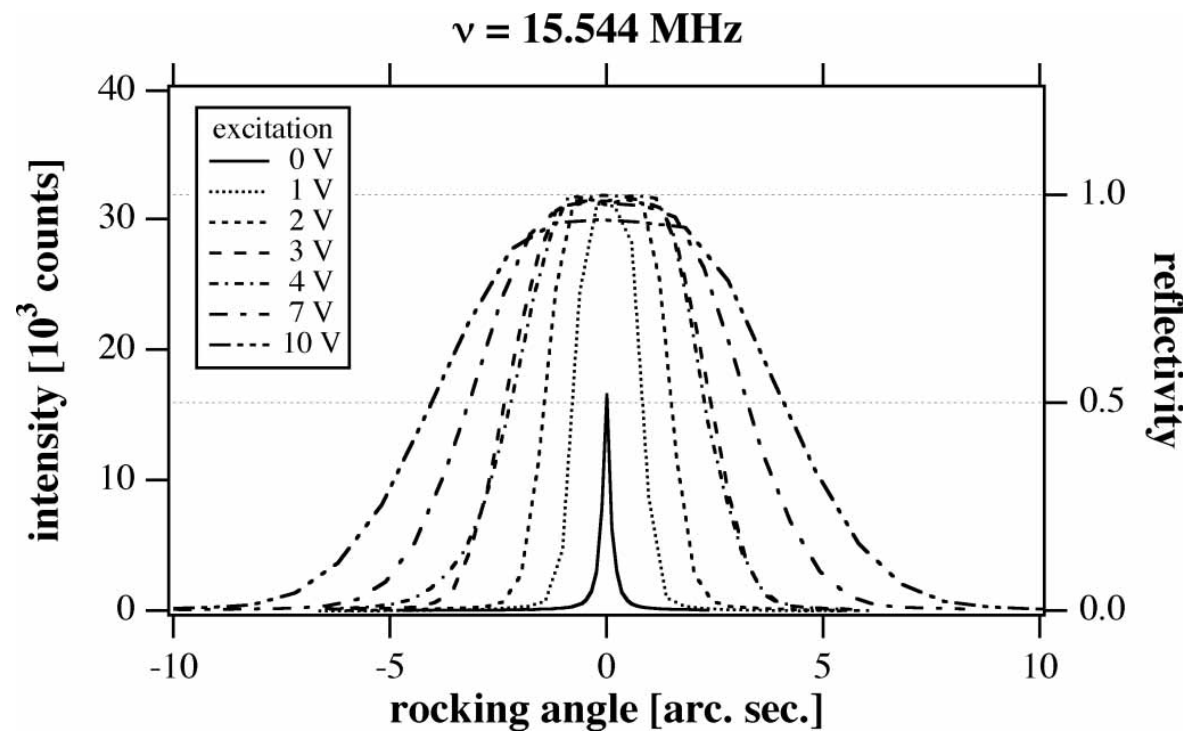

FIGURE 21 Evolution of rocking curves in a perfect crystal subjected to a low-frequency ultrasonic field. The central curve belongs to the unexcited crystal and is close to the intrinsic reflection curve as obtained by the dynamical theory of diffraction. An ultrasonic field first doubles the maximum intensity and then broadens the curve according to the lattice parameter distribution, i.e. the strain field in the crystal. From Liss et al. (1997b).

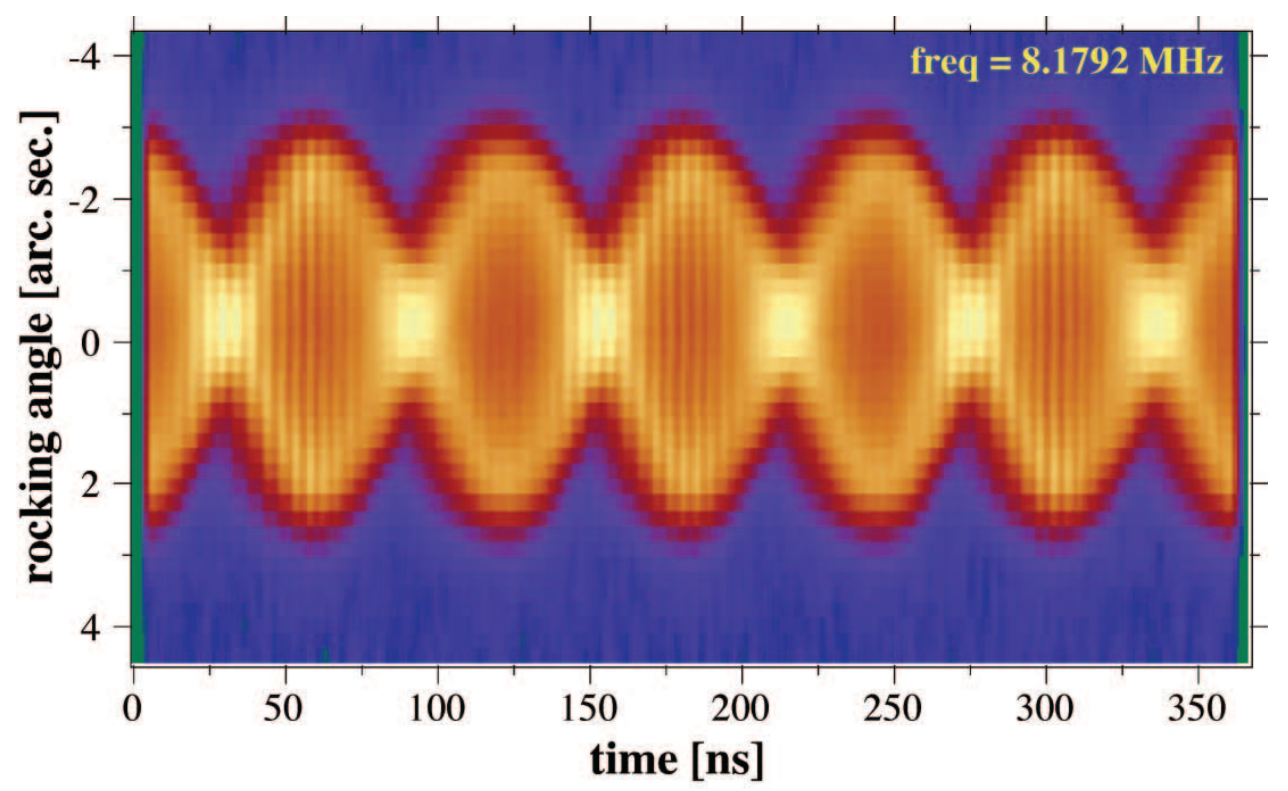

FIGURE 22 Time-resolved intensity distribution of a rocking curve such as presented in Fig. 21. The ultrasonic strain field occurs in form of a standing wave with zero-transitions, the nodes and maximal amplitude, the antinodes, as a function of time. Like a pendulum, the whole crystal volume oscillates here with a sinusoidal behavior. From Liss et al. (1998a). 


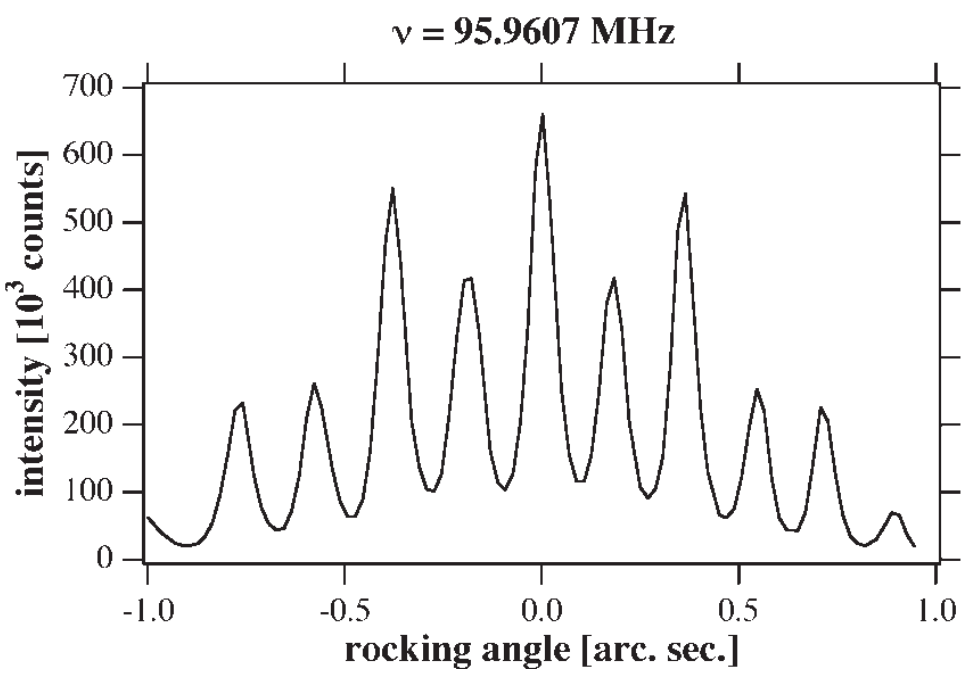

FIGURE 23 Diffraction pattern around the $\overline{7} 51$ reflection of a perfect silicon crystal excited by a highfrequency ultrasonic field along [111]. The wavelength of the longitudinal ultrasonic volume-wave is much shorter than the Pendellösung period, such that the diffraction process resolves the periodic modulation of the crystalline lattice revealing satellite peaks. Note the narrow scan range of only 2 arc sec and the resolution corresponding to about $10^{-7}$ of the triple-axes diffractometer in the transverse direction. Courtesy of Hock et al. (1999).

reflection. Satellites appear as shown in Fig. 23 (Hock et al., 1999). Their spacing is inverse proportional to the ultrasonic wavelength of about $10 \mu \mathrm{m}$ for this case. Thus at lower frequencies, they join too close together and overlap with the resolution function of the instrument. Not only the strain density distribution is obtained by the envelope function of the satellites but also detailed information of the wave field composition is obtained through the intensity modulation, i.e. the structure factor of the side reflections. In this sense, Fig. 23 shows two modes of excitation, one spaced with half the period compared to the other.

Changes in the crystallographic structure are of major importance in the characterization of phase transitions in solid state physics. Besides the studies cited above we present here examples on europium arsenide $\left(\mathrm{EuAs}_{3}\right)$ (Chattopadhyay et al., 1997) and manganese sulfide $\left(\mathrm{MnS}_{2}\right)$ which both order antiferromagnetically. Figures 24 and 25 show the lattice strain as a function of temperature. Decreasing from high temperatures, the lattice parameters shrink according to the usual phonon contribution in an anharmonic model. At the phase transitions, however, anomalies occur: there is a negative expansion in the case of $\mathrm{EuAs}_{3}$ where the lattice parameter increases with decreasing temperature. The behavior of the strain curve is very similar to the order parameter, which is proportional to the square root of the intensity of a pure magnetic reflection as in the inset of Fig. 24. Further evaluations of this similarity have been conducted on the system $\mathrm{NaV}_{2} \mathrm{O}_{5}$ (Chatterji et al., 1998). In the case of $\mathrm{MnS}_{2}$, the shrinking lattice parameter splits into two peaks below the phase transition, Fig. 25. The cubic lattice at high temperatures is tetragonally deformed along the axis of antiferromagnetic order and both peaks according to the $a$ - and $c$-axes are observed simultaneously by integration over several domains. 


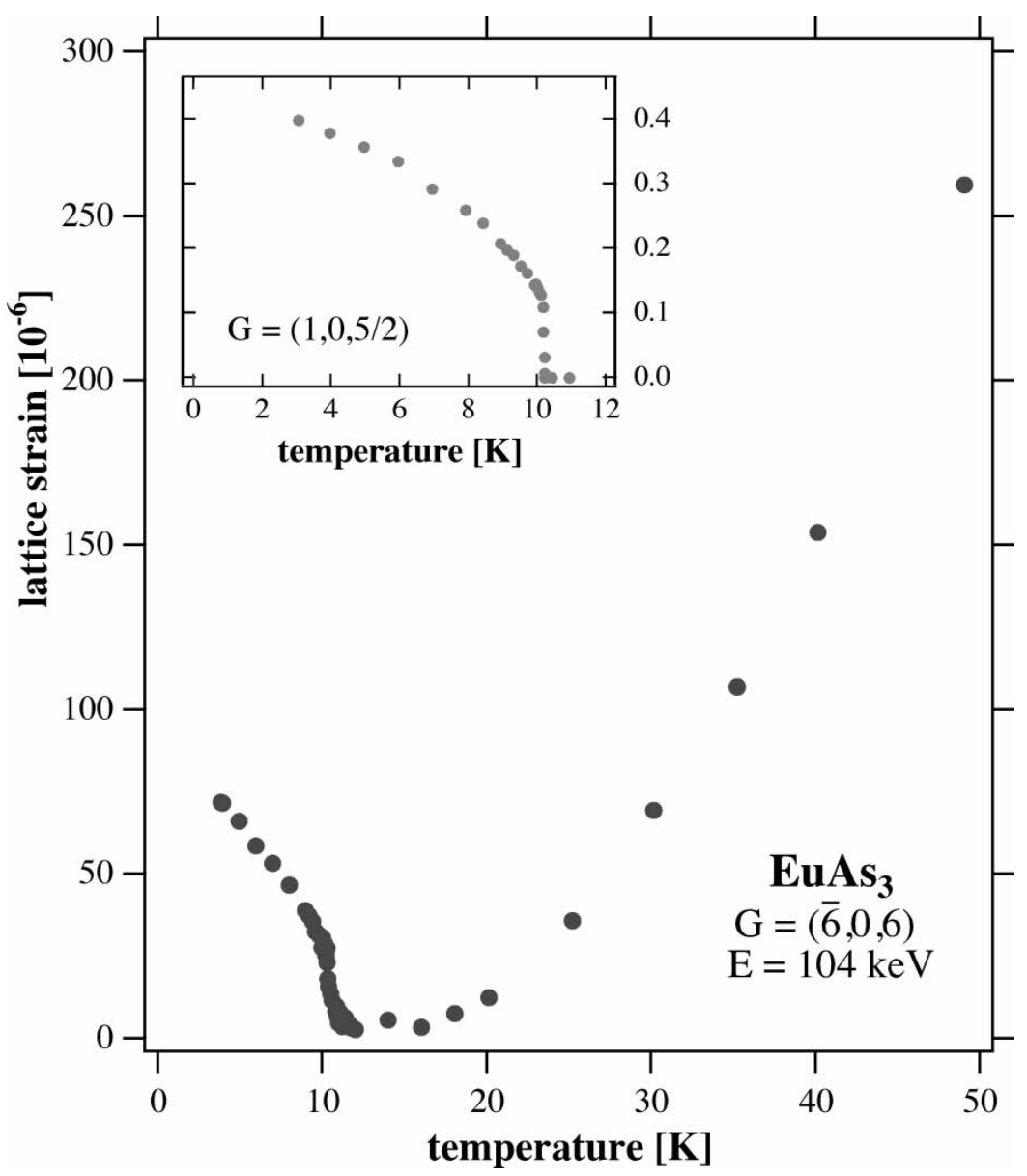

FIGURE 24 Thermal variation of the $\overline{6}, 0,6$ lattice spacing of EuAs 3 . The evolution for higher temperatures originates from phonon processes whereas the anomalous expansion below the phase transitions at 11 and $10.3 \mathrm{~K}$ is related to magnetic ordering. The latter is obtained by the sublattice magnetization measured by the integrated reflectivity of the nonresonant magnetic 1, 0,5/2 Bragg reflection shown as an inset. Courtesy of Chattopadhyay et al. (1997).

\section{CONCLUSION}

High-energy X-rays benefit from the high penetration power, the flat Ewald-sphere and the scattering mainly into the forward direction in order to be applied in various fields of solid state physics and materials science. Different techniques, such as the older and well established high resolution triple-crystal diffractometer, the newer two-dimensional detection setups and the energy dispersive methods have been developed during the last decade. Particularly, the employment of two-dimensional detectors with the possibility of parallel data acquisition requires attention to the resolution and data accuracy as presented here which can drive i.e. stress analysis to larger benefits. Future developments will have to combine the different techniques, such as the fast acquisition of a global reciprocal space map and the orientation matrix of a crystal with a two-dimensional detector together with a highest resolution scan with a triple crystal 


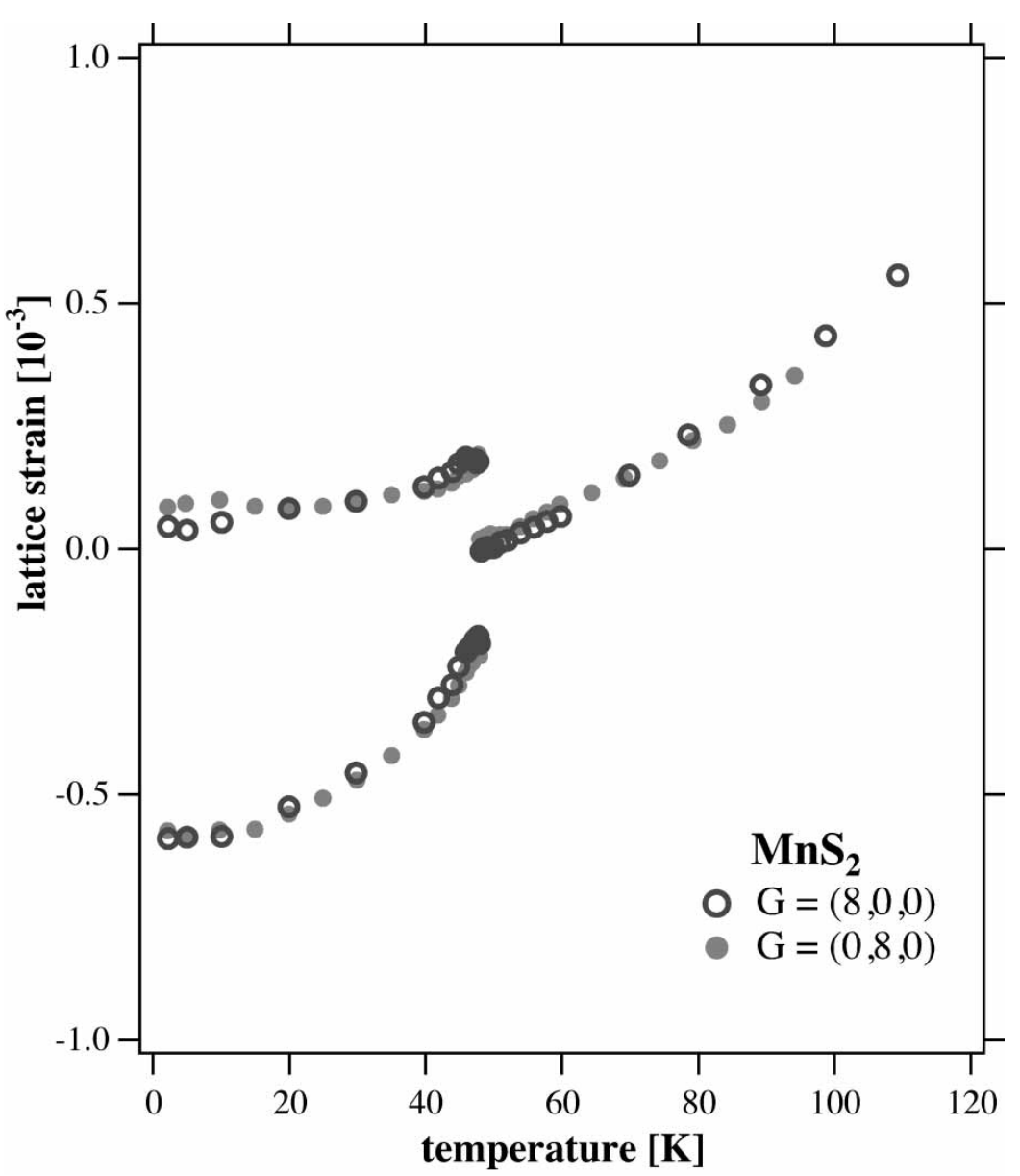

FIGURE 25 Thermal expansion of the lattice parameter of $\mathrm{MnS}_{2}$ measured at the $8,0,0$ and the $0,8,0$ reflections of the cubic system. Two values are observed below the magnetic phase transition at $48 \mathrm{~K}$ indicating a tetragonal deformation of the lattice. Courtesy of Chattopadhyay et al. (1997).

diffractometer. Further imaging and diffraction techniques have to be combined for parallel acquisition in topography and tomography. Huge potential is hidden in the time resolving techniques as there is enough intensity at third generation synchrotron sources in order to investigate kinetics and in-situ processes. Developments of faster detectors are on the way and are necessary for a further milestone in X-ray diffraction.

\section{Acknowledgments}

The authors of this review article wish to thank their partners who contributed to the examples presented in the different sections. In particular we appreciate and enjoyed the collaboration with Anke Pyzalla, Thomas Buslaps, Alain Jacques and Olivier Ferry for the energy dispersive results, Alexander Wanner, Jochen Böhm, Reinhard Kampmann, Thomas Lippmann and Hermann Franz for the strain measurements on TiAl, Michael Ohler and Agnes Royer for the crystal quality mapping, Rainer Hock and Andreas 
Magerl for the ultrasonic measurements and Tapan Chatterji for the works on antiferromagnetic materials. Further we like to thank the teams on ID15/ESRF and BW5/ PETRA-II/HASYLAB for their enthusiasm and instrumental support.

\section{References}

Arzel, L., Hehlen, B., Dénoyer, F., Currat, R., Liss, K.-D. and Courtens, E. (2003). Observation of a sample dependent $37 \mathrm{~K}$ anomaly on the lattice parameters of strontium titanate. Europhysics Letters, 61(5), 653-659.

Arzel, L., Hehlen, B., Tagantsev, A.K., Dénoyer, F., Liss, K.-D., Currat, R. and Courtens, E. (2002). Low-T ferroelectricity in antiphase domain boundaries of $\mathrm{SrTiO}_{3}$. Ferroelectrics, 267, 317-322.

Bartels, A., Kestler, H. and Clemens, H. (2002). Deformation behavior of differently processed gamma-titanium aluminides. Materials Science and Engineering, A(329-331C), 153-162.

Bartels, A., Schillinger, W., Graßl, G. and Clemens, H. (2003). Texture formation in $\gamma$-TiAl sheets. In: Kim, Y.-W., Clemens, H. and Rosenberger, A. (Eds.), Gamma Titanium Aluminides, TMS: Warrendale, PA, USA (in press).

Bastie, P., Dolino, G. and Hamelin, B. (2003). Opalescence of quartz: a study by high energy X-ray diffraction. Journal of Physics D: Applied Physics, 36, A139-A142.

Bastie, P., Hamelin, B. and Courtois, P. (2000). Méthode de Laue refocalisée à haute énergie : intérêts et apports de la spectrométrie des faisceaux diffractés. Journal de Physique, IV(10), Pr10_21-PR10_26.

Beckmann, F., Lippmann, T., Metge, J., Dose, T., Donath, T., Tischer, M., Liss, K.D. and Schreyer, A. (2003). HARWI-II, the new high-energy beamline for materials science at HASYLAB/DESY, In: SRI 2003. AIP Conference Proceedings, San Francisco (in press).

Berger, M.J., Hubbell, J.H., Seltzer, S.M., Coursey, J.S. and Zucker, D.S. (1998). XCOM: Photon Cross Sections Database. National Institute of Standards and Technology, http://physics.nist.gov/ PhysRefData/Xcom/Text/XCOM.html

Bikit, I., Slivka, J., Anicin, I.V., Marinkov, L. and Rudic, A. (1987). On the 'nonresonant' contribution to the photoactivation of 115-In-m. Zbornik Radova Prirodno-Matematickog Fakulteta, Serija za Fiziku, 17, 55-64.

Böhm, J., Wanner, A., Kampmann, R., Franz, H., Liss, K.-D., Schreyer, A. and Clemens, H. (2003). Internal stress measurements by high-energy synchrotron X-ray diffraction at increased specimen-detector distance. Nuclear Instruments and Methods in Physics Research, B(200), 315-322.

Bouchard, R., Hupfeld, D., Lippmann, T., Neuefeind, J., Neumann, H.B., Poulsen, H.F., Rutt, U., Schmidt, T., Schneider, J.R., Sussenbach, J. and von Zimmermann, M. (1998). A triple-crystal diffractometer for high-energy synchrotron radiation at the HASYLAB high-field wiggler beamline BW5. Journal of Synchrotron Radiation, 5, 90-101.

Bouchard, R., Kouptsidis, S., Neumann, H.-B., Schmidt, T. and Schneider, J.R. (1993). Nonimaging characterization of imperfect single-crystals by means of a 3-crystal diffractometer for high-energy synchrotron radiation. Journal of Applied Physics, 73(8), 3680-3684.

Broda, M., Pyzalla, A. and Reimers, W. (1999). X-ray analysis of residual stresse in C/SiC composites. Applied Composite Materials, 6(1), 51-66.

Brokmeier, H.-G., Günther, A., Yi, S.-B. and Ye, W. (2003). Investigation of local textures in extruded magnesium by synchrotron radiation. Advances in X-ray Analysis, 46, 151-156.

Brusch, G. (1998). Entwicklung eines Me $\beta$ - und Auswerteverfahrens zur Analyse von Eigenspannungsverteilungen mit Hochenergie-Röntgenbeugung. Dissertation, Hahn-Meitner-Institut, Berlin

Bunge, H.-J. and Klein, H. (1996). Determination of quantitative, high-resolution pole figures with the area detector. Zeitschrift für Metallkunde, 87(6), 465-475.

Chatterji, T., Liß K.-D., McIntyre, G. J., Weiden, M., Hauptmann, R. and Geibel, C. (1998). The ground state of $\mathrm{NaV}_{2} \mathrm{O}_{5}$. Solid State Communications, 108(1), 23-26.

Chattopadhyay, T., Liss, K.-D. and Brückel, T. (1997). Incommensurate-commensurate lock-in phase transition in EuAss. In: International Conference of Magnetism, Cairns, Australia.

Chavanne, J., Van Vaerenbergh, P. and Elleaume, P. (1999). A 3 T asymmetric permanent magnet wiggler. Nuclear Instruments \& Methods in Physics Research A, 421(1-2), 352-360.

Fischer, J.E., Milliken, J.W., Magerl, A., Kim, H.J. and Moore, A.W. (1991). Intercalated Graphite Neutron Optical-Components - Low-Mosaic Acceptor Compounds with Large D-Spacings. Nuclear Instruments \& Methods in Physics Research Section a-Accelerators Spectrometers Detectors and Associated Equipment, 300(1), 207-209.

Freund, A., Pynn, R., Stirling, W.G. and Zeyen, C.M.E. (1983). Vertically focussing Heusler alloy monochromators for polarized neutrons. Physica, B(120), 86-90. 
Goorsky, M.S., Yoon, H., Ohler, M. and Liss, K.-D. (1999). Defect depth profiling of CdZnTe using high-energy diffraction measurements. Proceedings SPIE, 3768, 108-114.

Günther, A., Brokmeier, H.-G., Petrovsky, E., Siemes, H., Helming, K. and Quade, H. (2002). Mineral preferred orientation and magnetic properties as indicators of varying strain conditions in naturally deformd iron ore. Applied Physics A, 74, 1080-1082.

Hahn, U., Schulte-Schrepping, H., Balewski, K., Schneider, J.R., Ilinski, P., Lai, B., Yun, W., Legnini, D. and Gluskin, E. (1997). Measurements of emittance and absolute spectral flux of the PETRA undulator at DESY Hamburg. Journal of Synchrotron Radiation, 4, 1-5.

Hatton, P. D., Wilkins, S. B., Spencer, P. D., v. Zimmermann, M., d'Almeida, T. (2003). High energy X-ray scattering studies of strongly correlated oxides. Journal of Physics D: Applied Physics, 36, A157-A161.

Helmers, L. (1996). Erstarrung stark seigender Zweistoffsysteme: Untersuchungen zum Bridgman-Verfahren an Mischungen der Elemente Germanium und Silizium. Dissertation, Rheinisch Westfälische Technische Hochschule Aachen

Hock, R., Magerl, A. and Liss, K.-D. (1999). To be Published.

Holland-Moritz, D., Schenk, T., Bellissent, R., Simonet, V., Funakoshi, K., Merino, J.M., Buslaps, T. and Reutzel, S. (2002). Short-range Order in Undercooled Co Melts. Journal of Non-Crystalline Solids, 312, 47-51.

Isaacs, E.D., Shukla, A., Platzman, P.M., Hamann, D.R., Barbiellini, B. and Tulk3 C. A. (1999). Covalency of the hydrogen bond in ice: a direct X-ray measurement. Physical Review Letters, 82(3), 600-603.

Jauch, W. (2001). High-quality structure factors: benefits from the use of gamma radiation. Journal of Physics and Chemistry of Solids, 62(12), 2103-2108.

Jauch, W. and Palmer, A. (1999). Anomalous zero-point-motion in SrTiO3: results from gamma-ray diffraction. Physical Review B, 60(5), 2961-2963.

Jolie, J., Materna, T., Masschaele, B., Mondelaers, W., Honkimaki, V., Koch, A. and Tschentscher, T. (1999). Heavy element sensitive tomography using Synchrotron Radiation above $100 \mathrm{keV}$. In: Speciation, Techniques and Facilities for Radioactive Materials at Synchrotron Light Sources, pp. 249-255, Nuclear Energy Agency, Grenoble.

Kampmann, R., Lippmann, T., Burmester, J., dos Santos, J. F., Franz, H., Haese-Seiler, M. and Marmotti, M. (2001). Upgrading of the PETRA-2 beamline at HASYLAB for materials science analyses. Nuclear Instruments \& Methods in Physics Research Section A, 467, 1261-1264.

Kestler, H., Clemens, H., Baur, H., Joos, R., Gerling, R., Cam, G., Bartels, A., Schleinzer, C. and Smarsly, W. (1999). Characterization of gamma TiAl sheet material for aeroengine application. In: Kim, Y.-W., Dimiduk, D.M. and Loretto, M.H. (Eds.), Gamma Titanium Aluminides II, pp. 423-430. TMS: Warrendale, PA, USA.

Lame, O., Bellet, D., Di Michiel, M. and Bouvard, D. (2003). In situ microtomography investigation of metal powder compacts during sintering. Nuclear Instruments \& Methods in Physics Research Section B, 200, 287-294.

Lippert, M., Brückel, T., Köhler, T. and Schneider, J. R. (1994). High resolution bulk magnetic scattering of high energy synchrotron radiation. Europhysics Letters, 27(7), 537-541.

Lippmann, T. and Schneider, J.R. (2000a). Topological analyses of cuprite, Cu2O, using high-energy synchrotron-radiation data. Acta Crystallographica Section A, 56, 575-584.

Lippmann, T. and Schneider, L.R. (2000b). Accurate structure-factor measurements using high-energy synchrotron radiation: a test on cuprite, Cu2O. Journal of Applied Crystallography, 33, 156-167.

Liß, K.-D. (1994). Strukturelle Charakterisierung und Optimierung der Beugungseigenschaften von $\mathrm{Si}_{1-x} G e_{x}$ Gradientenkristallen, die aus der Gasphase gezogen wurden. Dissertationsschrift, Rheinisch Westfälische Technische Hochschule Aachen

Liss, K.-D., Kaiser, M., Currat, R., Denoyer, F., Hlinka, J. and Hock, R. (2003). Kinetics of the field induced commensurate to ferroelectric phase transition in thiourea. Journal of Physics D: Applied Physics, 36, A172-A176.

Liss, K.-D., Magerl, A., Hock, R., Remhof, A. and Waibel, B. (1997a). Towards a new (Q, t) regime by time-resolved X-ray diffraction: ultra-sound excited crystals as an example. Europhysics Letters, 40(4), 369-374.

Liss, K.-D., Magerl, A., Hock, R., Waibel, B. and Remhof, A. (1998a). The investigation of ultrasonic fields by time resolved X-ray diffraction. Proceedings of SPIE, 3451, 117-127.

Liss, K.-D., Magerl, A., Remhof, A. and Hock, R. (1997b). Ultrasound induced gradient crystals observed by high energy X-rays. Acta Crystallographica, A(53), 181-186.

Liss, K.-D., Royer, A., Tschentscher, T., Suortti, P. and Williams, A.P. (1998b). On high resolution reciprocal space mapping with a triple crystal diffractometer for high energy X-rays. Journal of Synchrotron Radiation, 5, 82-89.

Madar, R., Mastromatteo, E., Magerl, A., Liss, K.-D. and Bernard, C. (1992). Low pressure chemical vapor deposition of massive $\mathrm{Si}_{1-x} \mathrm{Ge}_{x}$ gradient crystals and applications in short-wavelength diffraction. Surface and Coatings Technology, 54-55, 229-233.

Magerl, A., Liss, K.-D., Doll, C., Madar, R. and Steichele, E. (1994). Will gradient crystals become available for neutron diffraction?. Nuclear Instruments and Methods, A 338, 83-89. 
Magerl, A., Liss, K.-D., Hastings, J.B., Siddons, D.P., Neumann, H.-B., Poulsen, H.F., Rütt, U., Schneider, J.R. and Madar, R. (1995). The local perfection of massive gradient crystals studied by high-energy XRay diffraction. Europhysics Letters, 31(5-6), 329-334.

Maniguet, L., Bastie, P., Bellet, D. and Dupeux, M. (1995). Non-destructive bulk detection of localized straining by $\gamma$-ray diffractometry. Physica Status Solidi A, A(148), 389-406.

Marmotti, M., Burmester, J., Haese-Seiller, M. and Kampmann, R. (2002). Two-dimensional position-sensitive detectors for high resolution diffraction with neutrons and high energy synchrotron radiation. Nuclear Instruments \& Methods in Physics Research Section A, 477(1-3), 347-352.

Marques, M.A., Cabaco, M.I., Marques, M.I.D. and Gaspar, A.M. (2002). Intermediate-range order in aqueous solutions of salts constituted of divalent ions combined with monovalent counter-ions. Journal of Physics-Condensed Matter, 14(32), 7427-7448.

Materna, T., Jolie, J., Mondelaers, W., Masschaele, B., Honkimäki, V., Koch, A. and Tschentscher, T. (1999). Uranium-sensitive tomography with synchrotron radiation. Journal of Synchrotron Radiation, 6, 1059-1064.

May, C., Klimanek, P. and Magerl, A. (1995). Plastic bending of thin beryllium blades for neutron monochromators. Nuclear Instruments and Methods, A(357), 511-518.

Miclaus, C. and Goorsky, M.S. (2003). Strain evolution in hydrogen-implanted silicon. Journal of Physics D: Applied Physics, 36, A177-A180.

Neuefeind, J. and Liss, K.-D. (1996). Bond angle distribution in amorphous germania and silica. Berichte der Bunsengesellschaft für Physikalische Chemie, 100, 1341-1349.

Neumann, H.-B. (1991). Auflösungsvermögen eines dispersionsfreien Dreikristalldiffraktometers für hochenergetische Synchrotronstrahlung. Interner Bericht, 91-06, HASYLAB, DESY,

Neumann, H.-B., Poulsen, H.F., Rütt, U., Schneider, J.R. and Zimmermann, M.v. (1995). Investigation of structural phase transitions in perovskites using high energy synchrotron radiation. Phase Transitions, B55(1-4), 17-35.

Neumann, H.B., Rütt, U., Schneider, J.R. and Nagasawa, H. (1994). The resolution function of a triple crystal diffractometer for high energy synchrotron radiation in nondispersive Laue-geometry. Journal of Applied Crystallography, 27, 1030-1038.

Notthoff, C., Franz, H., Hanfland, M., Herlach, D.M., Holland-Moritz, D., Jacobs, G., Lippok, R., Petry, W. and Platzek, D. (1999). Energy dispersive X-ray diffraction on undercooled metallic melts. Journal of Non-Crystalline Solids, 252, 632-636.

Poulsen, H.F., Zimmermann, M.v., Schneider, J.R., Andersen, N.H., Schleger, P., Madsen, J., Hadfield, R., Casalta, H., Liang, R., Dosanjh, P. and Hardy, W. (1996). Structural phase transitions in bulk $\mathrm{Yba}_{2} \mathrm{Cu}_{3} \mathrm{O}_{6+x}$ with $x=0.35$ and $x=0.36$, Physical Review, B53(22), 15335-15344.

Pyzalla, A., Jacques, A., Feiereisen, J.-P., Buslaps, T., d'Almeida, T. and Liss, K.-D. (2001). In-situ analysis of the microstrains during tensile deformation of an AlSi-MMC at room temperature and elevated temperature. Journal of Neutron Research, 9, 435-442.

Pyzalla, A., Reimers, W. and Liss, K.-D. (2000a). A comparison of neutron and high energy synchrotron radiation as tools for texture and stress analysis. Materials Science Forum, 347-349, 34-39.

Pyzalla, A., Reimers, W., Royer, A. and Liss, K.-D. (1999). Microstructure, texture and residual stresses after cold extrusion - Application of white high energy synchrotron radiation. In: Proceedings of the 20th Riso International Symposium on Materials Science, pp. 453-458, Risø National Laboratory, Denmark.

Pyzalla, A., Wegener, J., Müller, K.B. and Liss, K.-D. (2000b). Influence of extrusion parameters on microstructure and texture of AlSi25Cu4Mg1. Materials Science Forum, 331-337, 781-786.

Pyzalla, A., Wegener, J., Müller, K.B. and Liss, K.-D. (2000c). Microstructure, texture and residual stresses of hot-extruded AlSi-alloys. In: Jouffrey, B. and Svejcar, J. (ed.), Microstructural Investigation and Analysis, Vol. 4, pp. 96-102. Wiley-VCH.

Rauch, H. and Petrascheck, D. (1976). Grundlagen für ein Laue-Neutroneninterferometer Teil 1: Dynamische Beugung, AIAU 74405b, Atominstitut der Österreichischen Universitäten,

Rauch, H. and Petrascheck, D. (1978). Dynamical neutron diffraction and its application. In: H. Dachs, (Ed.), Neutron Diffraction, pp. 303-351. Springer-Verlag, Berlin Heidelberg, New York.

Reichert, H., Bugaev, V.N., Shchyglo, O., Schops, A., Sikula, Y. and Dosch, H. (2001). Strain-induced nonanalytic short-range order in the spin glass $\mathrm{Cu}_{83} \mathrm{Mn}_{17}$. Physical Review Letters, 87(23), art. no.-236105.

Reimers, W., Broda, M., Brusch, G., Dantz, D., Liss, K.-D., Pyzalla, A., Schmakers, T. and Tschentscher, T. (1998). Evaluation of residual stresses in the bulk of materials by high energy synchrotron radiation. Journal of Nondestructive Evaluation, 17, 129-139.

Reimers, W., Pyzalla, A., Broda, M., Brusch, G., Dantz, D., Schmackers, T., Liss, K.D. and Tschentscher, T. (1999). The use of high-energy synchrotron diffraction for residual stress analyses. Journal of Materials Science Letters, 18, 581-583.

Rossberg, A., Piechotka, M., Magerl, A. and Kaldis, E. (1994). Mapping of the structural perfection of vapor grown $\alpha-\mathrm{HgI}_{2}$ crystals by means of $\gamma$-ray rocking curves. Journal of Applied Physics, 75(7), 3371-3376.

Royer, A. and Bastie, P. (1997). A non-destructive technique for the microstructural investigation of nickel base single crystal superalloys: high resolution diffraction of high energy synchrotron radiation. Scripta Materialia, 36(10), 1151-1159. 
Royer, A., Bastie, P. and Véron, M. (1997a). High energy X-ray diffraction measurement of the superstructure reflection (100) for a creep deformed AM1 single crystal superalloy specimen. Scripta Materialia, 37(8), 1199-1201.

Royer, A., Bastie, P. and Véron, M. (1997b). Temperature dependence of superstructure and fundamental reflections: study of a creep deformed single crystal nickel base superalloy AM1. Materials Science and Engineering, A(234-236), 1110-1113.

Rütt, U., Beno, M.A., Strempfer, J., Jennings, G., Kurtz, C. and Montano, P.A. (2001). Diffractometer for high energy X-rays at the APS. Nuclear Instruments \& Methods in Physics Research A, 467, 1026-1029.

Rütt, U., Neumann, H.-B., Poulsen, H.F. and Schneider, J.R. (1995). The resolution function of a triple-crystal diffractometer for high-energy synchrotron radiation. Journal of Applied Crystallography, 28, 729.

Schillinger, W., Lorenzen, B. and Bartels, A. (2002). Anisotropic mechanical behavior of textured $\gamma$-TiAl caused by the directionality of twinning. Materials Science and Engineering, A(329-331C), 641-645.

Schneider, J.R. (1974). A $\gamma$-ray diffractometer: a tool for investigating mosaic structure. Journal of Applied Crystallography, 7, 541-546.

Schnier, C. (2002). A new hypothesis for the existence of superheavy elements in the earth's crust. Journal of Radioanalytical and Nuclear Chemistry, 251(2), 191-196.

Schreyer, A., Lippmann, T., Beckmann, F., Metge, J., Kampmann, R., Dose, T., Liss, K.-D. and Tischer, M. (2003). HARWI II - The new beamline for materials science. Status Report, GKSS Forschungszentrum, http://genf.gkss.de/

Sohn, Y.U., Choi, J.Y., Kim, S.N., Kim, K.R., Bak, J.S. and Koo, Y.M. (2001). Hard X-ray beamline of 7.5T superconducting wiggler at Pohang light source. Nuclear Instruments \& Methods in Physics Research A, 467, 707-710.

Strempfer, J., Brückel, T., Hupfeld, D., Schneider, J.R., Liss, K.-D. and Tschentscher, T. (1997). The non resonant magnetic X-ray scattering cross section for photon energies up to $500 \mathrm{keV}$. Europhysics Letters, 40, 569-574.

Strempfer, J., Brückel, T., Rütt, U., Schneider, J.R., Liss, K.-D. and Tschentscher, T. (1996). The non resonant magnetic X-ray scattering cross section of $\mathrm{MnF}_{2}$. 2: High energy X-ray diffraction at $80 \mathrm{keV}$. Acta Crystallographica, A52, 438-449.

Suortti, P., Buslaps, T., Di Michiel, M., Honkimaki, V., Lienert, U., McCarthy, J.E., Merino, J.M. and Shukla, A. (2001a). Dispersion-compensating scanning X-ray spectrometer for Compton profile measurements. Nuclear Instruments \& Methods in Physics Research Section A, 467, 1541-1544.

Suortti, P., Buslaps, T., Honkimaki, V., Kretzschmer, M., Renier, M. and Shukla, A. (2001b). Monochromators for high-energy synchrotron radiation. Zeitschrift Fur Physikalische Chemie, 215, $1419-1435$.

Terry, A.E., Vaughan, G.B.M., Kvick, A., Walton, R.I., Norquist, A.J. and O'hare, D. (2002). In-situ timeresolved X-ray diffraction: the current state of the art. Synchrotron Radiation News, 15, 4-13.

Tomberli, B., Benmore, C.J., Egelstaff, P.A., Neuefeind, J. and Honkimäki, V. (2001). Temperature dependence of structural quantum effects in liquid methanol. Europhysics Letters, 55(3), 341-347.

Tschentscher, T. and Suortti, P. (1998). Experiments with very high energy synchrotron radiation. Journal of Synchrotron Radiation, 5, 286-292.

Wcislak, L., Bunge, H.J., Klein, H., Garbe, U. and Schneider, J.R. (2003). High-resolution texture imaging with hard synchrotron radiation in the moving area detector technique. Nuclear Instruments \& Methods in Physics Research Section B, 199, 38-43.

Wcislak, L., Schneider, J.R., Tschentscher, T., Klein, H. and Bunge, H.J. (2001). Hard X-ray texture measurements with an on-line image plate detector. Nuclear Instruments \& Methods in Physics Research Section A, 467, 1257-1260.

Wilkins, S.B., Hatton, P.D., Liss, K.-D., Ohler, M., Katsufuji, T. and Cheong, S.-W. (2000). High-resolution high energy X-ray diffraction studies of charge ordering in CMR manganites and nickelates. International Journal of Modern Physics B, 14(29, 30 and 31), 3753-3758.

Withers, P.J., Preuss, M., Webster, P.J., Hugues, D.J. and Korsunsky, A.M. (2002). Residual strain measurement by synchrotron diffraction. Materials Science Forum, 404-407, 1-12.

Zhukov, S.G., Chernyshev, V.V., Aslanov, L.A., Vakhrushev, S.B. and Schenk, H. (1995). X-ray analysis and computer modeling of the structure of 'relaxor' ferroelectrics $\mathrm{Pb} 3 \mathrm{MgNb} 2 \mathrm{O} 9$ and $\mathrm{Pb} 2 \mathrm{ScTaO} 6$ in the paraelectric state. Journal of Applied Crystallography, 28, 385-391. 